\title{
Beamforming Optimization for Intelligent Reflecting Surface Aided SWIPT IoT Networks Relying on Discrete Phase Shifts
}

\author{
Shiqi Gong, Ziyi Yang, Chengwen Xing, Jianping An, and Lajos Hanzo, Fellow, IEEE
}

\begin{abstract}
Intelligent reflecting surface (IRS) is capable of constructing the favorable wireless propagation environment by leveraging massive low-cost reconfigurable reflectarray elements. In this paper, we investigate the IRS-aided MIMO simultaneous wireless information and power transfer (SWIPT) for Internet of Things (IoT) networks, where the active base station (BS) transmit beamforming and the passive IRS reflection coefficients are jointly optimized for maximizing the minimum signal-tointerference-plus-noise ratio (SINR) among all information decoders (IDs), while maintaining the minimum total harvested energy at all energy receivers (ERs). Moreover, the IRS with practical discrete phase shifts is considered, and thereby the max-min SINR problem becomes a NP-hard combinatorial optimization problem with a strong coupling among optimization variables. To explore the insights and generality of this maxmin design, both the Single-ID Single-ER (SISE) scenario and the Multiple-IDs Multiple-ERs (MIME) scenario are studied. In the SISE scenario, the classical combinatorial optimization techniques, namely the special ordered set of type 1 (SOS1) and the reformulation-linearization (RL) technique, are applied to overcome the difficulty of this max-min design imposed by discrete optimization variables. Then the optimal branch-and-bound algorithm and suboptimal alternating optimization algorithm are respectively proposed. We further extend the idea of alternating optimization to the MIME scenario. Moreover, to reduce the iteration complexity, a two-stage scheme is considered aiming to separately optimize the BS transmit beamforming and the IRS reflection coefficients. Finally, numerical simulations demonstrate the superior performance of the proposed algorithms over the benchmarks in both the two scenarios.
\end{abstract}

Index Terms-Alternating optimization, combinatorial opti-

Manuscript received August 12, 2020; revised November 09, 2020; accepted December 15, 2020. This work was supported by the National Key Research and Development Program of China under No. 2019YFB1803200, in part by the State Key Laboratory of Rail Traffic Control and Safety (Contract No. RCS2018K005), Beijing Jiaotong University, in part by the National Natural Science Foundation of China under Grants U1836201, 61722104, 61671058, and 61620106001 . L. Hanzo would like to acknowledge the financial support of the Engineering and Physical Sciences Research Council projects EP/N004558/1, EP/P034284/1, EP/P034284/1, EP/P003990/1 (COALESCE), of the Royal Society's Global Challenges Research Fund Grant as well as of the European Research Council's Advanced Fellow Grant QuantCom. The associate editor coordinating the review of this paper and approving it for publication was Dr. Nicola Marchetti. (Corresponding author: Chengwen Xing)

S. Gong, Z. Yang, C. Xing, and J. An are with the School of Information and Electronics, Beijing Institute of Technology, Beijing 100081, China. S. Gong is also with the State Key Laboratory of Rail Traffic Control and Safety, Beijing Jiaotong University, Beijing 100044, China (e-mails: gsqyxyx@gmail.com, yangziyi18@163.com, xingchengwen@gmail.com and an@bit.edu.cn).

Lajos Hanzo is with the School of Electronics and Computer Science, University of Southampton, SO17 1BJ, UK (e-mail: 1h@ecs.soton.ac.uk).

Copyright (c) 20xx IEEE. Personal use of this material is permitted. However, permission to use this material for any other purposes must be obtained from the IEEE by sending a request to pubs-permissions@ieee.org. mization, discrete phase shifts, intelligent reflecting surface (IRS), simultaneous wireless information and power transfer (SWIPT).

\section{INTRODUCTION}

The explosive growth in the number of intelligent communication devices and the proliferation of data hungry wireless applications both require higher spectral and energy efficiencies of Internet-of-Things (IoT) networks. Although the massive multiple-input multiple-output (MIMO) technology is capable of significantly improving the efficiency of both wireless information transfer (WIT) and wireless power transfer (WPT) in emerging IoT networks by exploiting the large array gain, this is usually achieved at high capital expense [1]-[3]. As a remedy, it is possible to use much less number of radio frequency (RF) chains than transmit/receive antennas under a so-called hybrid implementation, which may also result in high hardware cost, high signal processing overhead and high energy consumption of IoT networks, thereby hampering its practical implementation. As a cost-effective alternative to the massive MIMO technology, intelligent reflecting surface (IRS) is capable of achieving unprecedented spectral and energy efficiencies, especially in complex propagation scenarios suffering from severe blockage. However, since IRS is in essence a reconfigurable metal surface equipped with a large number of passive reflecting elements, it cannot perform sophisticated signal processing as the large-scale array and the active MIMO relay, and is usually deployed with low hardware cost and low power consumption. In practice, by adjusting the phase shift and amplitude attenuation (jointly referred to as reflection coefficients) of each IRS reflecting element, a favorable wireless propagation environment for both WIT and WPT is proactively reconstructed [4], [5].

Given the above benefits, the research on IRS-aided communications for various wireless systems, such as MISO systems [6], [7], point-to-point MIMO systems [8], multicell multiuser MIMO systems [9] and MIMO-OFDM systems [10], [11], have drawn an upsurge of attention. These studies usually assume the perfect channel state information (CSI) of the considered IRS-aided wireless systems for characterizing the performance limits. In practice, since the IRS operated without the RF chains lacks the baseband processing capability and a large number of IRS-related channels need to be estimated, the traditional training-based channel estimation scheme is not directly applicable. As an alternative, various cascaded transmitter-IRS-receiver channel estimation schemes using the 
IRS grouping strategy were proposed for both frequencyflat and frequency-selective channels under the assumption of uplink-downlink channel reciprocity [10]-[13].

As a parallel trend, a novel radio frequency (RF) energy harvesting technique has been conceived for overcoming the energy scarcity issue of IoT networks, considering limited energy supplies provided by traditional wire cables and batteries [14], [15]. Nevertheless, the new challenges arise in integrating the RF energy harvesting and the advanced WIT techniques for realizing sustainable green IoT networks. To this end, simultaneous wireless information and power transfer (SWIPT) has been evaluated as an appealing and innovative technology [16]. Recently, there have been growing concerns regarding the IRS-aided SWIPT systems with the assumption of perfect CSI [9], [17]-[19]. For example, Wu et. al [17] investigated the weighted harvested energy maximization in an IRS-aided MISO SWIPT system and demonstrated that the dedicated energy beamforming is actually unnecessary. As a further development, the maximization of the minimum harvested energy among all energys receivers (ERs) in this system was investigated from a fairness perspective [18]. By deploying multiple IRSs, Wu et. al [19] further investigated the total transmit power minimization subject to individual QoS constraints at both information decoders (IDs) and ERs. Pan et.al [9] considered more general IRS-aided MIMO SWIPT systems and studied the weighted sum rate maximization of all IDs, while guaranteeing a certain minimum total harvested energy at all ERs. A variety of advanced communication techniques in IoT networks, such as non-orthogonal multiple access (NOMA) [20], [21], physical layer security [22], [23] and mobile edge computing (MEC), have also been integrated with the IRS to achieve better system performance.

In terms of our work, we consider a IRS-aided MIMO SWIPT system that consists of one multi-antenna base station (BS), one IRS assisting communication and multiple IoT devices supporting SWIPT. In particular, two types of IoT devices are assumed, i.e. multiple single-antenna IDs and multiple multi-antenna ERs, and the IRS is deployed for assisting the SWIPT from the BS to these IoT devices. From the fairness perspective, we further investigate the maximization of the minimum SINR among all IDs by jointly optimizing the active BS transmit beamforming vectors and the passive IRS reflection coefficients (also referred to as the IRS reflection matrix) subject to the minimum required total harvested energy at all ERs. In contrast to the above IRS related studies with the assumption of continuous phase shifts, our work considers the realistic finite-resolution IRS phase shifts. In other words, the phase shift of each IRS reflecting element is restricted to a finite number of discrete values [13], [24]. Nonetheless, since the BS transmit beamforming vectors and the IRS discrete phase shifts are strongly coupled in both the objective function and constraints of the max-min SINR problem, this combinatorial optimization problem is generally nonconvex and NP-hard, to which the derivation of the globally optimal solution is still an open challenge.

To the best of our knowledge, this paper presents the first comprehensive attempt to study the max-min fairness of the IRS-aided MIMO SWIPT system with realistic finite-

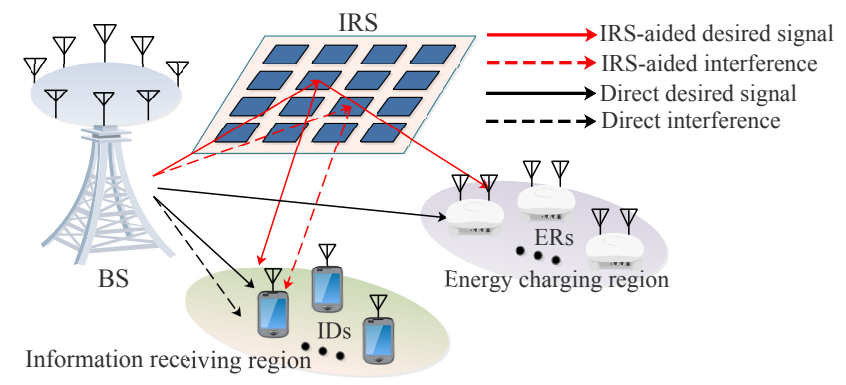

Fig. 1. An IRS-aided MIMO SWIPT system.

resolution phase shifts. Firstly, we consider the single-ID and single-ER (SISE) scenario, in which the max-min SINR problem is simplified to the signal-to-noise ratio (SNR) maximization problem. To tackle this intractable problem, we firstly investigate its feasibility and the tightness of the predefined energy harvesting constraint using the well-known combinatorial optimization techniques. Specifically, for the optimization of IRS discrete phase shifts, this intractable maxmin SINR problem can be modeled as a mixed-integer nonlinear programming (MINLP) problem via the special ordered set of type 1 (SOS1) [25], and then the reformulation-linearization (RL) technique is applied to reformulate this MINLP problem as a classical 0-1 linear integer problem (LIP) [26]. Armed with the above transformations, the optimal branch and bound algorithm and the suboptimal alternating optimization algorithm are proposed, respectively, depending on the tightness of the energy harvesting constraint. Furthermore, to avoid high complexity of solving the LIP for a large number of IRS reflecting elements, we propose a low-complexity alternative algorithm using the element-by-element iterative strategy.

Secondly, we consider the general scenario of multiple IDs and multiple ERs (MIME), in which both the feasibility and tightness analysis of the max-min SINR problem follow the same methodology as that in the SISE scenario. In this scenario, the intractable max-min SINR problem can be equivalently transformed into a power minimization problem, motivated by the fact that the optimal transmit power is monotonically non-decreasing with respect to the minimum SINR among all IDs. We then propose jointly applying the bisection search and an alternating optimization algorithm to find a high-quality suboptimal solution to the original optimization problem. To mitigate the computational burden of this two-layer iterative optimization, we also develop a lowcomplexity two-stage scheme for independently optimizing the BS transmit beamforming vectors and the IRS discret phase shifts. Finally, numerical experiments further demonstrate the excellent output SNR and minimum SINR performance of our proposed algorithms in the SISE and MIME scenarios, respectively.

The rest of this paper is organized as follows. Section II introduces the system model and the problem formulation for the IRS-aided MIMO SWIPT system. Section III mainly studies the SISE scenario, where the feasibility and tightness analysis of the optimization problem are firstly involved, and then the optimal algorithm and suboptimal alternating optimization algorithms are proposed respectively depending on the tightness of the energy harvesting constraint. Section IV extends the idea of alternating optimization to the MIME 
scenario, and also introduces a low-complexity two-stage scheme. Section V presents numerical results to demonstrate the excellent performance of the proposed algorithms. Finally, Section VI concludes this paper.

Notations: Vectors and matrices are denoted by the boldfaced lower-case and upper-case letters, respectively. $(\cdot)^{*},(\cdot)^{\mathrm{T}}$, $(\cdot)^{\mathrm{H}}$ and $(\cdot)^{-1}$ denote the conjugate, transpose, Hermitian and inverse of a matrix, respectively. $\boldsymbol{A} \succeq \mathbf{0}$ means that the square matrix $\boldsymbol{A}$ is positive semidefinite. $\operatorname{Pha}(\boldsymbol{a})$ and $\Re\{\boldsymbol{a}\}$ represents the element-wise angle and real-part extraction of a complex vector $\boldsymbol{a}$, respectively. $\operatorname{Tr}(\boldsymbol{A}),\|\boldsymbol{a}\|$ and $|a|$ denote the trace of a matrix, the Euclidean norm of a vector and the absolute value of a complex scalar $a$, respectively. $\operatorname{diag}(\boldsymbol{A})$ denotes a vector whose elements are the diagonal elements of $\boldsymbol{A}$. while $\operatorname{diag}\left(\left[a_{1}, \cdots, a_{N}\right]\right)$ denotes a diagonal matrix whose diagonal elements consist of a $N$-dimensional vector $\boldsymbol{a}=\left[a_{1}, \cdots, a_{N}\right] . \boldsymbol{A}[i, j]$ and $\boldsymbol{a}[i]$ denote the ith row and jth column element of $\boldsymbol{A}$ and the $i$ th element of $\boldsymbol{a}$, respectively. $\mathbb{E}[\boldsymbol{A}]$ and $\operatorname{vec}(\boldsymbol{A})$ denote the statistical expectation operation and vectorization operation on a matrix $\boldsymbol{A}$ (where all columns of $\boldsymbol{A}$ are stacked on top of each other ), respectively. $\boldsymbol{A} \otimes \boldsymbol{B}$ is the Kronecker matrix product. $\mathcal{C N}\left(\mu, \sigma^{2}\right)$ stands for a circularly symmetric complex Gaussian variable with mean $\mu$ and variance $\sigma^{2}$. The words 'independent and identically distributed' and 'with respect to' are abbreviated as 'i.i.d.' and 'w.r.t.', respectively.

\section{System Model ANd PROBlem Formulation}

\section{A. An IRS-Aided MIMO SWIPT System}

As shown in Fig. 1, we consider an IRS-aided MIMO SWIPT system composed of an $N_{S}$-antenna BS, an IRS equipped with $N_{R}$ passive reflecting elements, denoted by the set $\mathcal{N}=\left\{1, \cdots, N_{R}\right\}$, and two types of IoT devices aiming for harvesting energy and receiving information, respectively. Specifically, the IRS is deployed to assist SWIPT from the BS to $K_{E}$ multi-antenna ERs and $K_{I}$ single-antenna IDs, denoted by the sets $\mathcal{K}_{\mathcal{I}}=\left\{1, \cdots, K_{I}\right\}$ and $\mathcal{K}_{E}=\left\{1, \cdots, K_{E}\right\}$, respectively. Note that each ER is equipped with $N_{U}$ antennas for scavenging more RF energy to be used for future information transfer. Moreover, with the aid of a smart controller, IRS can dynamically adjust the reflection coefficients of all elements for implementing the favorable wireless propagation environment [7]. Regardless of nonlinear hardware imperfections and circuit noise, the ideal IRS reflection coefficients are modeled as $\alpha_{n}=a_{n} e^{j \theta_{n}}, \forall n \in \mathcal{N}$, where $\left|a_{n}\right| \leq 1$ and $\theta_{n}$ denote the amplitude and phase shift of the $n$th IRS reflecting element, respectively. For ease of practical circuit implementation, we assume that $a_{n}=1$ for maximizing the reflected signal strength, and the phase shift $\theta_{n}$ of each IRS reflecting element can only take a finite number of values ranging from 0 to $2 \pi$. Specifically, we define $b$ as the number of bits devoted to uniformly quantizing the interval $[0,2 \pi)$, thereby forming a set of IRS discrete phase shifts denoted by $\mathcal{P}=\{0, \Delta \theta, \cdots,(L-1) \Delta \theta\}$ with $\Delta \theta=\frac{2 \pi}{L}$ and cardinality $L=2^{b}$.

As demonstrated in [17], there is no need for the BS to transmit any dedicated energy signal for improving energy harvesting performance of ERs ${ }^{1}$. Therefore, we assume that the BS only transmits information signals to serve both IDs and ERs. Based on this fact, the BS transmitted signal is given by $\boldsymbol{s}=\sum_{i_{I}=1}^{K_{I}} \boldsymbol{v}_{i_{I}} s_{i_{I}}$, where $s_{i_{I}} \sim \mathcal{C N}(0,1), \forall i_{I} \in \mathcal{K}_{I}$ denotes the Gaussian signal for ID $i_{I}$ and $\boldsymbol{v}_{i_{I}} \in \mathbb{C}^{N_{S} \times 1}$ is the associated beamforming vector. The maximum transmit power at the BS is then given by $\sum_{i_{I} \in \mathcal{K}_{I}}\left\|\boldsymbol{v}_{i_{I}}\right\|^{2} \leq P_{0}$. Furthermore, let's define $\boldsymbol{h}_{d, i_{I}}^{H} \in \mathbb{C}^{1 \times N_{S}}\left(\boldsymbol{H}_{d, i_{E}} \in \mathbb{C}^{N_{U} \times N_{S}}\right)$, $\boldsymbol{G} \in \mathbb{C}^{N_{R} \times N_{S}}$ and $\boldsymbol{h}_{r, i_{I}}^{H} \in \mathbb{C}^{1 \times N_{R}}\left(\boldsymbol{H}_{r, i_{E}} \in \mathbb{C}^{N_{U} \times N_{R}}\right)$ as the baseband equivalent channels from the BS to ID $i_{I}$ (ER $i_{E}$ ), from the BS to the IRS, and from the IRS to ID $i_{I}$ (ER $i_{E}$ ), respectively. All channel matrices above consider the joint effects of large-scale path loss and small-scale fading. We also assume frequency-flat quasi-static downlink channels and the uplink-downlink channel reciprocity. Moreover, all signal processing tasks are considered to be carried out at the BS, and the BS is capable of acquiring perfect CSI using the pilot-assisted channel estimation and feedback over a fading coherence block. Under these assumptions, our work actually characterizes the fairness performance limit of the considered IRS-aided SWIPT IoT network. ${ }^{2}$ Specifically, by combining the signal received directly from the BS and that reflected by the IRS, the received signal at ID $i_{I}$ is then given by

$$
y_{i_{I}}=\boldsymbol{h}_{i_{I}}^{H} \boldsymbol{s}+n_{i_{I}}=\sum_{j_{I} \in \mathcal{K}_{I}} \boldsymbol{h}_{i_{I}}^{H} \boldsymbol{v}_{j_{I}} s_{j_{I}}+n_{i_{I}}, \quad \forall i_{I} \in \mathcal{K}_{I}
$$

where $\boldsymbol{h}_{i_{I}}^{H}=\boldsymbol{h}_{d, i_{I}}^{H}+\boldsymbol{h}_{r, i_{I}}^{H} \boldsymbol{\Phi} \boldsymbol{G}$ denotes the compound downlink channel from the BS to ID $i_{I}$. The diagonal matrix $\boldsymbol{\Phi}=$ $\operatorname{diag}\left[\alpha_{1}, \cdots, \alpha_{N_{R}}\right]$ consists of $N_{R}$ reflection coefficients of the IRS. $n_{i_{I}} \sim \mathcal{C N}\left(\mathbf{0}, \sigma_{i_{I}} \boldsymbol{I}_{N_{D}}\right)$ represents the additive white Gaussian noise (AWGN) at ID $i_{I}$. It follows from (1) that the SINR of ID $i_{I}$ is expressed as

$$
\operatorname{SINR}_{i_{I}}=\frac{\left|\boldsymbol{h}_{i_{I}}^{H} \boldsymbol{v}_{i_{I}}\right|^{2}}{\sum_{j_{I} \neq i_{I}}\left|\boldsymbol{h}_{i_{I}}^{H} \boldsymbol{v}_{j_{I}}\right|^{2}+\sigma_{i_{I}}^{2}}, \quad \forall i_{I} \in \mathcal{K}_{I},
$$

Similarly, by neglecting the sufficiently small noise power, the harvested RF power at ER $i_{E}$ is given by

$$
E_{i_{E}}=\eta_{i_{E}} \sum_{i_{I} \in \mathcal{K}_{I}}\left\|\boldsymbol{H}_{i_{E}} \boldsymbol{v}_{i_{I}}\right\|^{2}, \quad \forall i_{E} \in \mathcal{K}_{E},
$$

where $\boldsymbol{H}_{i_{E}}=\boldsymbol{H}_{d, i_{E}}+\boldsymbol{H}_{r, i_{E}} \boldsymbol{\Phi} \boldsymbol{G}$ and $0<\eta_{i_{E}} \leq 1$ denotes the energy harvesting efficiency at ER $i_{E}$.

\section{B. Problem Formulation}

In our work, we jointly optimize the BS transmit beamforming vectors $\boldsymbol{v}_{i_{I}}$ 's and the IRS reflection coefficients $\alpha_{n}$ 's

\footnotetext{
${ }^{1}$ It is worth noting that by following the same methodology as that in the later Proposition 3, our considered max-min SINR problem (P1) subject to the total harvested energy constraint at all ERs (where the active energy threshold $E_{0} \in\left[E_{0}^{\mathrm{mi}}, E_{0}^{\max }\right]$ is assumed) can be equivalently transformed into the total harvested energy maximization problem (P1) in [17] subject to individual SINR constraints at all IDs.

${ }^{2}$ More practically, most existing works aim to estimate the cascaded BSIRS-user channels using the IRS grouping strategy (as introduced in Section I), based on which the estimated group BS-IRS-user channels generally have much smaller dimension [11], [12]. Under this channel estimation framework, the corresponding robust design complexity of the IRS reflection matrix is also much reduced. However, this topic is beyond the scope of our current work, and we will consider it as the future research direction.
} 
to maximize the minimum SINR among all IDs, while guaranteeing the minimum required harvested energy at all ERs. The corresponding optimization problem is formulated as

$$
\begin{aligned}
(\mathrm{P} 1): \max _{\left\{\boldsymbol{v}_{i_{I}}\right\}, \boldsymbol{\Phi}} & \min _{i_{I} \in \mathcal{K}_{I}} \operatorname{SINR}_{i_{I}} \\
\text { s.t. } & \sum_{i_{I} \in \mathcal{K}_{I}}\left\|\boldsymbol{v}_{i_{I}}\right\|^{2} \leq P_{0}, \boldsymbol{\Phi}=\operatorname{diag}\left[\alpha_{1}, \cdots, \alpha_{N_{R}}\right], \\
& \sum_{i_{E} \in \mathcal{K}_{E}} \sum_{i_{I} \in \mathcal{K}_{I}} \eta_{i_{E}}\left\|\boldsymbol{H}_{i_{E}} \boldsymbol{v}_{i_{I}}\right\|^{2} \geq E_{0}, \\
& \operatorname{Pha}\left(\alpha_{n}\right) \in \mathcal{P}, \quad \forall n \in \mathcal{N},
\end{aligned}
$$

where $E_{0}$ denotes the minimum total harvested energy threshold. Since the IRS reflection coefficients $\alpha_{n}$ 's are coupled with the BS transmit beamforming vectors $\boldsymbol{v}_{i_{I}}$ 's, problem (P1) is in essence an NP-hard combinatorial optimization problem, and the globally optimal solution is hard to obtain. A popular suboptimal approach is to temporarily omit the limitation of IRS discrete phase shifts, and then directly map the obtained continuous phase shifts to the nearest discrete values in $\mathcal{P}$ one by one [27]. Unfortunately, this suboptimal scheme may have poor inter-user interference suppression capability when the low-resolution IRS phase shifts are considered, and thereby is not pursued in our work. On the other hand, problem (P1) can also be equivalently transformed into a quadratic assignment problem (QAP) by using SOS1 [25]. However, due to the tightly coupled continuous and binary variables, it is still challenging to derive the optimal solution of problem (P1).

\section{SISE SCENARIO}

In this section, we mainly consider the SISE scenario, i.e. $K_{I}=K_{E}=1$, which can be regarded as a practical scenario where multiple IDs and multiple ERs use the orthogonal multiple access technique for communicating with the BS, thereby resulting in no interference among IDs. In this context, we firstly perform feasibility and tightness analysis of problem (P1) using the classical combinatorial optimization techniques, where the optimal branch-and-bound algorithm and the suboptimal alternating optimization algorithm are proposed for the inactive and active energy harvesting constraints, respectively. To further alleviate the computational burden, we also consider a low-complexity alternating optimization algorithm using the element-by-element iterative strategy.

\section{A. Feasibility Analysis}

Firstly, in the SISE scenario, problem (P1) is simplified to ${ }^{3}$

$$
\begin{aligned}
\max _{\boldsymbol{v}_{I}, \boldsymbol{\Phi}} & \left|\boldsymbol{h}_{I}^{H} \boldsymbol{v}_{I}\right|^{2} \\
\text { s.t. } & \left\|\boldsymbol{v}_{I}\right\|^{2} \leq P_{0}, \quad \eta_{E}\left\|\boldsymbol{H}_{E} \boldsymbol{v}_{I}\right\|^{2} \geq E_{0}, \\
& \boldsymbol{\Phi}=\operatorname{diag}\left[\alpha_{1}, \cdots, \alpha_{N_{R}}\right], \quad \operatorname{Pha}\left(\alpha_{n}\right) \in \mathcal{P}, \quad \forall n \in \mathcal{N} .
\end{aligned}
$$

It is obvious that problem (5) is much easier to solve than problem (P1) due to the much reduced coupling among optimization variables. Motivated by the minimum harvested energy threshold $E_{0}$, we next study the feasibility of problem (5) to explore the inherent structure of the optimal solution

\footnotetext{
${ }^{3}$ In this section, the subscripts $i_{I}$ and $i_{E}$ are abbreviated as $I$ and $E$, respectively, since only a single ID and a single ER are considered.
}

to problem (5). To be specific, the maximum feasible energy threshold $E_{0}^{\max }$ of problem (5) is determined such that

$$
\begin{aligned}
\max _{\boldsymbol{v}_{I}, \boldsymbol{\Phi}} & \eta_{E}\left\|\boldsymbol{H}_{E} \boldsymbol{v}_{I}\right\|^{2}=\eta_{1} \boldsymbol{v}_{I}^{H} \boldsymbol{H}_{E}^{H} \boldsymbol{H}_{E} \boldsymbol{v}_{I} \\
\text { s.t. } & \left\|\boldsymbol{v}_{I}\right\|^{2} \leq P_{0}, \\
& \boldsymbol{\Phi}=\operatorname{diag}\left[\alpha_{1}, \cdots, \alpha_{N_{R}}\right], \operatorname{Pha}\left(\alpha_{n}\right) \in \mathcal{P}, \forall n \in \mathcal{N} .
\end{aligned}
$$

For any given $\boldsymbol{\Phi}$, problem (6) is in essence an eigenvalue optimization problem w.r.t $\boldsymbol{v}_{I}$, whose optimal solution is obtained as $\boldsymbol{v}_{I}^{\star}=\sqrt{P_{0}} \boldsymbol{\mu}_{\max }\left(\boldsymbol{H}_{E}^{H} \boldsymbol{H}_{E}\right)$, where $\boldsymbol{\mu}_{\max }(\boldsymbol{A})$ denotes the unit-norm eigenvector of matrix $\boldsymbol{A}$ associated with its maximum eigenvalue $\lambda_{\max }(\boldsymbol{A})$ [28]. Further, substituting the obtained $\boldsymbol{v}_{I}^{\star}$ into problem (6) yields

$$
\begin{aligned}
\max _{\mathbf{\Phi}} & \eta_{E} P_{0} \lambda_{\max }\left(\boldsymbol{H}_{E}^{H} \boldsymbol{H}_{E}\right) \\
\text { s.t. } & \boldsymbol{\Phi}=\operatorname{diag}\left[\alpha_{1}, \cdots, \alpha_{N_{R}}\right], \quad \operatorname{Pha}\left(\alpha_{n}\right) \in \mathcal{P}, \forall n \in \mathcal{N} .
\end{aligned}
$$

Since the relationship between $\lambda_{\max }\left(\boldsymbol{H}_{E}^{H} \boldsymbol{H}_{E}\right)$ with $\boldsymbol{H}_{E}=$ $\boldsymbol{H}_{d, E}+\boldsymbol{H}_{r, E} \boldsymbol{\Phi} \boldsymbol{G}$ and $\boldsymbol{\Phi}$ is not analytical, the globally optimal solution to problem (7) can only be obtained by an exhaustive search on all possible combinations of IRS discrete phase shifts in $\mathcal{P}$, which has the worst-case complexity $\mathcal{O}\left(L^{N_{R}}\right)$. However, considering that an exhaustive search is computationally prohibitive for a large $N_{R}$ (i.e. when $N_{R} \geq 30$ and $L \geq 2$, we have $L^{N_{R}} \geq 10^{9}$ ), we propose an alternating optimization algorithm to obtain a locally optimal solution of problem (7). To be specific, we redefine $\boldsymbol{H}_{r, E}=\left[\boldsymbol{h}_{r, E}^{1}, \cdots, \boldsymbol{h}_{r, E}^{N_{R}}\right]$ and $\boldsymbol{G}=\left[\boldsymbol{g}_{1}, \cdots, \boldsymbol{g}_{N_{R}}\right]^{H}$ with $\boldsymbol{h}_{r, E}^{n} \in \mathbb{C}^{N_{U} \times 1}$ and $\boldsymbol{g}_{n} \in \mathbb{C}^{N_{S} \times 1}, \forall n \in \mathcal{N}$, then problem (7) can be rewritten in an explicit form of $\alpha_{n}=[\boldsymbol{\Phi}]_{n, n}$ as

$$
\begin{array}{cl}
\max _{\alpha_{n}} & \eta_{E} P_{0} \lambda_{\max }\left(\boldsymbol{A}_{n}+\alpha_{n} \boldsymbol{B}_{n}+\alpha_{n}^{*} \boldsymbol{B}_{n}\right), \\
\text { s.t. } & \operatorname{Pha}\left(\alpha_{n}\right) \in \mathcal{P}, \quad \forall n \in \mathcal{N} .
\end{array}
$$

where

$$
\begin{aligned}
\boldsymbol{A}_{n} & =\left(\boldsymbol{H}_{d, E}+\sum_{m=1, m \neq n}^{N_{R}} \alpha_{m} \boldsymbol{h}_{r, E}^{m} \boldsymbol{g}_{m}^{H}\right)\left(\boldsymbol{H}_{d, E}+\sum_{m=1, m \neq n}^{N_{R}} \alpha_{m} \boldsymbol{h}_{r, E}^{m} \boldsymbol{g}_{m}^{H}\right)^{H} \\
& +\left\|\boldsymbol{g}_{n}\right\|^{2} \boldsymbol{h}_{r, E}^{n}\left(\boldsymbol{h}_{r, E}^{n}\right)^{H} \\
\boldsymbol{B}_{n} & =\boldsymbol{h}_{r, E}^{n} \boldsymbol{g}_{n}^{H}\left(\boldsymbol{H}_{d, E}+\sum_{m=1, m \neq n}^{N_{R}} \alpha_{m} \boldsymbol{h}_{r, E}^{m} \boldsymbol{g}_{m}^{H}\right)^{H}
\end{aligned}
$$

The optimal $\alpha_{n}$ to problem (8) with given $\left\{\alpha_{m}\right\}_{m=1, m \neq n}^{N_{R}}$ is further calculated as

$$
\alpha_{n}^{\star}=\underset{\operatorname{Pha}(\alpha) \in \mathcal{P}}{\arg \max } \lambda_{\max }\left(\boldsymbol{A}_{n}+\alpha \boldsymbol{B}_{n}+\alpha^{*} \boldsymbol{B}_{n}\right), \forall n \in \mathcal{N} .
$$

By iteratively updating $\alpha_{n}$ according to (10) while holding $\left\{\alpha_{m}\right\}_{m=1, m \neq n}^{N_{R}}$ fixed, the objective value of problem (7) is guaranteed to be monotonically non-decreasing within the iterations, which is also upper-bounded by a finite maximum harvested energy corresponding to the IRS continuous phase shifts. Since problem (8) is usually solved exactly with the unique optimal solution shown in (10), by referring to [29, Theorem 2], we can conclude that every limit point generated by this alternating optimization algorithm with the worstcase complexity $\mathcal{O}\left(I_{A} L N_{R}\right)$, where $I_{A}$ denotes the number of iterations (all $N_{R}$ IRS discrete phase shifts are updated 
at each iteration), is a locally optimal (Boulingand stationary) solution of problem (7), since the discrete non-convex constraints are imposed on the IRS reflection coefficients [30]. In particular, numerical simulations in Section $\mathrm{V}$ will verify that this suboptimal algorithm achieves comparable performance to an exhaustive search by choosing the solution achieving the maximum objective value from a set of candidate solutions obtained from different random initializations. Once the optimal $\boldsymbol{\Phi}$ of problem (7) is obtained, we have $E_{0}^{\max }=\eta_{E} P_{0} \lambda_{\max }\left(\boldsymbol{H}_{E}^{\star H} \boldsymbol{H}_{E}^{\star}\right)$. Correspondingly, the feasible region of the energy threshold in problem (5) is given by $E_{0} \in\left[0, E_{0}^{\max }\right]$.

\section{B. Tightness Analysis}

Additionally, we investigate the tightness of the energy harvesting constraint in problem (5) as follows. By temporarily neglecting this constraint, problem (5) reduces to

$$
\begin{aligned}
\max _{\boldsymbol{v}_{I}, \boldsymbol{\Phi}} & \left|\boldsymbol{h}_{I}^{H} \boldsymbol{v}_{I}\right|^{2} \\
\text { s.t. } & \left\|\boldsymbol{v}_{I}\right\|^{2} \leq P_{0}, \quad \boldsymbol{\Phi}=\operatorname{diag}\left[\alpha_{1}, \cdots, \alpha_{N_{R}}\right], \\
& \operatorname{Pha}\left(\alpha_{n}\right) \in \mathcal{P}, \quad \forall n \in \mathcal{N} .
\end{aligned}
$$

Given any $\boldsymbol{\Phi}$, problem (11) has a similar structure to problem (6), and thereby the optimal $\boldsymbol{v}_{I}^{\star}=\sqrt{P_{0}} \boldsymbol{h}_{I} /\left\|\boldsymbol{h}_{I}\right\|$ can be derived, based on which problem (11) is simplified to

$$
\begin{aligned}
\max _{\boldsymbol{\Phi}} & P_{0}\left\|\boldsymbol{h}_{d, I}^{H}+\boldsymbol{h}_{r, I}^{H} \boldsymbol{\Phi} \boldsymbol{G}\right\|^{2}, \\
\text { s.t. } & \boldsymbol{\Phi}=\operatorname{diag}\left[\alpha_{1}, \cdots, \alpha_{N_{R}}\right], \operatorname{Pha}\left(\alpha_{n}\right) \in \mathcal{P}, \forall n \in \mathcal{N} .
\end{aligned}
$$

In contrast to problem (7), the objective function of problem (12) is in an explicit quadratic form of $\boldsymbol{\Phi}$. Moreover, using the special ordered set of type 1 (SOS1) which is defined as a set of binary vectors with at most one element being non-zero, i.e. a vector $\boldsymbol{x} \in \mathbb{C}^{L \times 1}$ satisfying $\sum_{l=1}^{L} \boldsymbol{x}[l]=1$ and $\boldsymbol{x}[l] \in\{0,1\}, \forall l \in \mathcal{L}$ with $\mathcal{L}=\{1, \cdots, L\}$, we can simply re-express the $n$th IRS reflection coefficient $\alpha_{n}$ as $\alpha_{n}=\boldsymbol{a}^{T} \boldsymbol{x}_{n}, \forall n \in \mathcal{N}$, where $\boldsymbol{a}=\left[1, e^{j \Delta \theta}, \cdots, e^{j \Delta(L-1) \theta}\right]^{T}$ consists of all possible discrete phase shifts, and thus the IRS reflection matrix $\boldsymbol{\Phi}$ is rewritten as $\boldsymbol{\Phi}=\operatorname{diag}\left(\left(\boldsymbol{I}_{N_{R}} \otimes \boldsymbol{a}^{T}\right) \boldsymbol{x}\right)$ with $\boldsymbol{x}=\left[\boldsymbol{x}_{1}^{T}, \cdots, \boldsymbol{x}_{N_{R}}^{T}\right]^{T}$, based on which problem (12) is transformed into the following 0-1 QAP (where all constant terms in the objective function are dropped)

$$
\begin{array}{ll}
\max _{\boldsymbol{x}} & \boldsymbol{x}^{T} \boldsymbol{C} \boldsymbol{x}+2 \Re\left\{\boldsymbol{x}^{T} \boldsymbol{d}\right\} \\
\text { s.t. } & \boldsymbol{x}=\left[\boldsymbol{x}_{1}^{T}, \cdots, \boldsymbol{x}_{N_{R}}^{T}\right]^{T}, \sum_{l=1}^{L} \boldsymbol{x}_{n}[l]=1, \boldsymbol{x}_{n}[l] \in\{0,1\} \\
\forall l \in \mathcal{L}, \forall n \in \mathcal{N},
\end{array}
$$

where $\boldsymbol{C}=\left(\operatorname{diag}\left(\boldsymbol{h}_{r, I}^{H}\right) \boldsymbol{G} \boldsymbol{G}^{H} \operatorname{diag}\left(\boldsymbol{h}_{r, I}\right)\right) \otimes\left(\boldsymbol{a a ^ { H }}\right) \in \mathbb{C}^{L N_{R} \times L N_{R}}$ and $\boldsymbol{d}=\operatorname{vec}\left(\boldsymbol{a} \boldsymbol{h}_{d, I}^{T}\left(\operatorname{diag}\left(\boldsymbol{h}_{r, I}^{H}\right) \boldsymbol{G}\right)^{T}\right) \in \mathbb{C}^{L N_{R} \times 1}, \forall n \in \mathcal{N}$. Inspired by the range invariance of the product of two binary variables, we consider applying the well-known RL technique to re-express this non-convex QAP as a 0-1 LIP which can be optimally solved by the standard branch-and-bound algorithm [25]. The following proposition presents this equivalent reformulation.
Proposition 1. Problem (13) can be equivalently linearized as the following 0-1 LIP whose globally optimal solution is derived by the standard branch-and-bound algorithm.

$$
\begin{aligned}
& \max _{\boldsymbol{x}, \boldsymbol{y}} \overline{\boldsymbol{d}}^{T} \boldsymbol{x}+\overline{\boldsymbol{c}}^{T} \boldsymbol{y}, \\
& \text { s.t. } \sum_{l=1}^{L} \boldsymbol{y}_{k(n, l, m, q)}=\boldsymbol{x}_{m}[q], \forall m, n \in \mathcal{N}, n<m, \forall q \in \mathcal{L}, \quad(14 \mathrm{~b}) \\
& \sum_{q=1}^{L} \boldsymbol{y}_{k(n, l, m, q)}=\boldsymbol{x}_{n}[l], \forall m, n \in \mathcal{N}, n<m, \forall l \in \mathcal{L}, \quad(14 \mathrm{c}) \\
& \sum_{l=1}^{L} \boldsymbol{x}_{n}[l]=1, \boldsymbol{x}_{n}[l] \in\{0,1\}, \boldsymbol{y}_{k(n, l, m, q)} \in\{0,1\}, \forall l, q \in \mathcal{L}, \\
& \forall n, m \in \mathcal{N},
\end{aligned}
$$

where $\overline{\boldsymbol{d}}=2 \Re\{\boldsymbol{d}\}+\operatorname{diag}(\boldsymbol{C})$ and $\overline{\boldsymbol{c}} \in \mathbb{C}^{K \times 1}$ with $K=$ $\frac{L^{2} N_{R}\left(N_{R}-1\right)}{2}$. The vector $\overline{\boldsymbol{c}}$ is related to $\boldsymbol{C}$ in a relatively complex manner. Specifically, the index $k(n, l, m, q)(1 \leq$ $k(n, l, m, q) \leq K$ ) of $\bar{c}$ is defined as

$$
\begin{aligned}
k(n, l, m, q) & =\frac{(n-1)\left(2 N_{R}-n\right)+2(m-n-1)}{2} L^{2} \\
& +(l-1) L+q,
\end{aligned}
$$

based on which the $k(n, l, m, q)$ th element of $\bar{c}$ is given by $\overline{\boldsymbol{c}}[k(n, l, m, q)]=\boldsymbol{C}[\hat{i}, \hat{j}]+\boldsymbol{C}[\hat{j}, \hat{i}]$, where $\hat{i}=l+(n-1) L$ and $\hat{j}=q+(m-1) L$. Note that the formulation (15) shows an analytical expression of the index $k(n, l, m, q)$ of $\overline{\boldsymbol{c}}(\boldsymbol{y})$ associated with the indices of both sets $\mathcal{L}$ and $\mathcal{N}$.

Proof. Let's recall problem (13) (equivalent to problem (12)), its involved integer constraints imply

$$
\begin{aligned}
& \boldsymbol{x}^{T} \boldsymbol{C} \boldsymbol{x}=\sum_{m, n, q, l} C_{n l m q} \boldsymbol{x}_{n}[l] \boldsymbol{x}_{m}[q]=\sum_{m, n, q, l, m \neq n} C_{n l m q} \boldsymbol{x}_{n}[l] \boldsymbol{x}_{m}[q] \\
& +\sum_{n, l} C_{n l n l} \boldsymbol{x}_{n}[l], \forall m, n \in \mathcal{N}, \forall q, l \in \mathcal{L} .
\end{aligned}
$$

where $C_{n l m q}$ denotes a particular element indexed by $\{n, l, m, q\}$ in $\boldsymbol{C}$. Since $\boldsymbol{x}_{m}[q] \boldsymbol{x}_{n}[l]=\boldsymbol{x}_{n}[l] \boldsymbol{x}_{m}[q]$, we only consider substituting variables $\left\{\boldsymbol{x}_{m}[q] \boldsymbol{x}_{n}[l]\right\}$ with $n<m$ for the sake of reducing the size of the newly introduced variables. Using the RL technique, for $\forall m, n \in \mathcal{N}, n<m, \forall q, l \in \mathcal{L}$, the term $\boldsymbol{x}_{m}[q] \boldsymbol{x}_{n}[l]$ can be replaced by a new binary variable $\boldsymbol{y}_{k(n, l, m, q)}$ satisfying $\boldsymbol{y}_{k(n, l, m, q)} \leq \boldsymbol{x}_{m}[q], \boldsymbol{y}_{k(n, l, m, q)} \leq \boldsymbol{x}_{n}[l]$ and $\boldsymbol{y}_{k(n, l, m, q)} \geq \boldsymbol{x}_{m}[q]+\boldsymbol{x}_{n}[l]-1$, where $k(n, l, m, q)$ is the index of vector $\boldsymbol{y} \in \mathbb{C}^{\frac{L^{2} N_{R}\left(N_{R}-1\right)}{2}}$. Correspondingly, the matrix $\boldsymbol{C}$ can also be stacked into a column vector $\overline{\boldsymbol{c}}$ using $\overline{\boldsymbol{c}}[k(n, l, m, q)]=\boldsymbol{C}[\hat{i}, \hat{j}]+\boldsymbol{C}[\hat{j}, \hat{i}]$ and the mapping rule in (15). As a result, the objective function of problem (13) can be rewritten as (14a).

Furthermore, we explore the implicit constraints on $\boldsymbol{y}_{k(n, l, m, q)}$ 's. Specifically, by multiplying both sides of $\sum_{l=1}^{L} \boldsymbol{x}_{n}[l]=1$ by $\boldsymbol{x}_{m}[q]$, we can obtain the constraint (14b) which also implies $\boldsymbol{y}_{k(n, l, m, q)} \leq \boldsymbol{x}_{m}[q]$. Similarly, the constraint $(14 \mathrm{c})$ can be obtained by multiplying both sides of $\sum_{l=1}^{L} \boldsymbol{x}_{m}[q]=1$ by $\boldsymbol{x}_{n}[l]$, and $\boldsymbol{y}_{k(n, l, m, q)} \leq \boldsymbol{x}_{m}[q]$ is also hinted. Finally, combining (14b) and (14c) further yields 


$$
\begin{aligned}
& \boldsymbol{x}_{m}[q]+\boldsymbol{x}_{n}[l]-1=\sum_{l^{\prime}=1}^{L} \boldsymbol{y}_{k\left(n, l^{\prime}, m, q\right)}+\boldsymbol{x}_{n}[l]-1 \\
& =\boldsymbol{x}_{m}[q] \boldsymbol{x}_{n}[l]+\sum_{l^{\prime} \neq l} \boldsymbol{x}_{m}[q] \boldsymbol{x}_{n}\left[l^{\prime}\right]+\boldsymbol{x}_{n}[l]-1 \\
& =\boldsymbol{x}_{m}[q] \boldsymbol{x}_{n}[l]+\sum_{l^{\prime} \neq l}\left(\boldsymbol{x}_{m}[q]-1\right) \boldsymbol{x}_{n}\left[l^{\prime}\right] \leq \boldsymbol{x}_{m}[q] \boldsymbol{x}_{n}[l] \\
& =\boldsymbol{y}_{k(n, l, m, q)}
\end{aligned}
$$

According to the above analysis, it is clear that the QAP (13) can be equivalently transformed into the LIP (14) by introducing an auxillary vector $\boldsymbol{y}$. This completes the proof.

Although the branch-and-bound algorithm used for solving the LIP (14) has the worst-case exponential complexity $\mathcal{O}\left(L^{N_{R}}\right)$, it will be shown to be effective for systems of smallto-medium scale (i.e. $N_{R} \leq 30$ and $L \leq 4$ ) via numerical simulations in Section V. Notice that in each branching process, the relaxed linear programming counterpart of problem (14) is solved at the cost of complexity $\mathcal{O}\left(\left(K+L N_{R}\right)^{3} L_{c}^{0.5}+\right.$ $\left.\left(K+L N_{R}\right)^{2} L_{c}^{1.5}\right)$ [31]. Furthermore, assuming the optimal $\boldsymbol{x}$ to problem (14), the optimal objective value of problem (12) based on $\boldsymbol{\Phi}=\operatorname{diag}\left(\left(\boldsymbol{I}_{N_{R}} \otimes \boldsymbol{a}^{T}\right) \boldsymbol{x}\right)$ is in fact an upper bound on that of problem (5), and the resultant harvested energy is expressed as $E_{0}^{\mathrm{mi}}=\eta_{E} P_{0}\left\|\boldsymbol{H}_{E} \boldsymbol{h}_{I}\right\|^{2} /\left\|\boldsymbol{h}_{I}\right\|^{2}$ with $\boldsymbol{H}_{E}=\boldsymbol{H}_{d, E}+\boldsymbol{H}_{r, E} \boldsymbol{\Phi} \boldsymbol{G}$ and $\boldsymbol{h}_{I}^{H}=\boldsymbol{h}_{d, I}^{H}+\boldsymbol{h}_{r, I}^{H} \boldsymbol{\Phi} \boldsymbol{G}$. It is readily inferred that for all $E_{0} \in\left[0, E_{0}^{\mathrm{mi}}\right)$, the energy harvesting constraint can be automatically satisfied at the optimal $\left\{\boldsymbol{v}_{I}, \boldsymbol{\Phi}\right\}$ to problem (11). In other words, the constraint in problem (5) is inactive when $E_{0} \in\left[0, E_{0}^{\mathrm{mi}}\right)$ and can be ignored without loss of optimality. However, when $E_{0} \in\left[E_{0}^{\mathrm{mi}}, E_{0}^{\max }\right]$ is considered, we have the following lemma.

Lemma 1. When $E_{0} \in\left[E_{0}^{\mathrm{mi}}, E_{0}^{\max }\right]$, the energy harvesting constraint is active at the optimal solution $\left\{\boldsymbol{v}_{I}^{\star}, \boldsymbol{\Phi}^{\star}\right\}$ of problem (5), which implies $\eta_{E}\left\|\boldsymbol{H}_{E}^{\star} \boldsymbol{v}_{I}^{\star}\right\|^{2}=E_{0}$.

Proof. Please see Appendix A.

In a nutshell, for the case of $E_{0} \in\left[0, E_{0}^{\mathrm{mi}}\right)$, problem (5) can be globally solved using the classical combinatorial optimization techniques, i.e. the SOS1 and the RL technique. Furthermore, when $E_{0} \in\left[E_{0}^{\mathrm{mi}}, E_{0}^{\max }\right]$ is considered, it is readily inferred that both the transmit power constraint and the energy harvesting constraint are active at the optimal solution, which thus makes problem (5) particularly challenging. In this context, we propose an alternating optimization algorithm to find a locally optimal solution of problem (5) by iteratively optimizing $\boldsymbol{v}_{I}$ and $\boldsymbol{\Phi}$. Firstly, for any given $\boldsymbol{\Phi}$, the optimal $\boldsymbol{v}_{I}$ to problem (5) is shown in the following Proposition.

Proposition 2. For any $E_{0} \in\left[E_{0}^{\mathrm{mi}}, E_{0}^{\max }\right]$, the globally optimal $\boldsymbol{v}_{I}$ to problem (5) with the given $\boldsymbol{\Phi}$ has the following form $\boldsymbol{v}_{I}^{\star}=\sqrt{P_{0}} \boldsymbol{\mu}_{\max }\left(\boldsymbol{h}_{I} \boldsymbol{h}_{I}^{H}+\beta^{\star} \eta_{E} \boldsymbol{H}_{E}^{H} \boldsymbol{H}_{E}\right)$, where $\beta^{\star}$ denotes the optimal dual variable corresponding to the energy harvesting constraint, and can be found by the subgradient method [32].

Proof. Please see Appendix B.

Using the $\boldsymbol{v}_{I}$ obtained from Proposition 2, we next investigate the subproblem w.r.t. $\mathbf{\Phi}$. Specifically, by jointly utilizing the SOS1 and the RL technique, the quadratic energy harvesting constraint $\eta_{1}\left\|\boldsymbol{H}_{E} \boldsymbol{v}_{I}\right\|^{2} \geq E_{0}$ can be linearized in the form of $\overline{\boldsymbol{d}}^{T} \boldsymbol{x}+\overline{\boldsymbol{c}}^{T} \boldsymbol{y}$, similarly to the reformulation of problem (12) shown in Proposition 1. As such, the subproblem w.r.t. $\Phi$ can also be modeled as a LIP and thus optimally solved by the standard branch-and-bound algorithm.

\section{Low-Complexity Alternating Optimization}

Generally, when the number of IRS reflecting elements is pretty large (i.e. $N_{R} \geq 36$ ), applying the RL technique to problem (5) usually faces a large computational burden due to the high dimension of the auxillary binary variable $\boldsymbol{y}$ in the LIP (14). For example, when $N_{R}=36$ and $L=2^{b}=4$ are adopted, the dimension of $\boldsymbol{y}$ becomes $K=\frac{L^{2} N_{R}\left(N_{R}-1\right)}{2} \approx 10^{4}$. which generally implies a high time complexity of the optimal LIP solution. As an alternative, a low-complexity alternating optimization algorithm is applied to find a locally optimal $\Phi$ to problem (5) by iteratively optimizing each of the IRS reflection coefficients (i.e. $\alpha_{n}$ ), while holding the others (i.e. $\left.\left\{\alpha_{m}\right\}_{m=1, m \neq n}^{N_{R}}\right)$ fixed. To be specific, for the case of $E_{0} \in$ $\left[0, E_{0}^{\mathrm{mi}}\right)$, problem (5) reduces to problem (12). Let's define $\boldsymbol{h}_{r, I}^{H}=\left[h_{r, I}^{1}, \cdots, h_{r, I}^{N_{R}}\right]$, the objective function of problem (12) can be rewritten in the following explicit form w.r.t. $\alpha_{n}$.

$$
P_{0}\left\|\boldsymbol{h}_{d, I}^{H}+\boldsymbol{h}_{r, I}^{H} \boldsymbol{\Phi} \boldsymbol{G}\right\|^{2}=A_{n}+\alpha_{n} B_{n}+\alpha_{n}^{*} B_{n}^{*}, \quad \forall n \in \mathcal{N},
$$

where

$$
\begin{aligned}
A_{n} & =P_{0}\left(\boldsymbol{h}_{d, I}^{H}+\sum_{m=1, m \neq n}^{N_{R}} \alpha_{m} h_{r, I}^{m} \boldsymbol{g}_{m}^{H}\right)\left(\boldsymbol{h}_{d, I}^{H}+\sum_{m=1, m \neq n}^{N_{R}} \alpha_{m} h_{r, I}^{m} \boldsymbol{g}_{m}^{H}\right)^{H} \\
& +P_{0}\left|h_{r, I}^{n}\right|^{2}\left\|\boldsymbol{g}_{n}\right\|^{2} \\
B_{n} & =P_{0} h_{r, I}^{n} \boldsymbol{g}_{n}^{H}\left(\boldsymbol{h}_{d, I}^{H}+\sum_{m=1, m \neq n}^{N_{R}} \alpha_{m} h_{r, I}^{m} \boldsymbol{g}_{m}^{H}\right)^{H}, \forall n \in \mathcal{N} . \text { (19) }
\end{aligned}
$$

Assuming $B_{n}=\left|B_{n}\right| e^{j \psi_{n}}$, it then follows from (18) that the optimal $\alpha_{n}$ to problem (12) with any given $\left\{\alpha_{m}\right\}_{m=1, m \neq n}^{N_{R}}$ is given by

$$
\alpha_{n}=\underset{\operatorname{Pha}(\alpha) \in \mathcal{P}}{\arg \max } \cos \left(\operatorname{Pha}(\alpha)+\psi_{n}\right), \quad \forall n \in \mathcal{N} .
$$

Furthermore, when $E_{0} \in\left[E_{0}^{\mathrm{mi}}, E_{0}^{\max }\right]$ is considered, it is easily inferred from Lemma 1 that compared to the case of $E_{0} \in\left[0, E_{0}^{\mathrm{mi}}\right)$, the optimization of the IRS reflection matrix $\boldsymbol{\Phi}$ needs to additionally satisfy the energy harvesting constraint due to its tightness at the optimal solution. Under the overall framework of the proposed alternating optimization algorithm for this case, we alternatively consider applying the elementby-element iterative strategy to solve the subproblem w.r.t. $\boldsymbol{\Phi}$. Specifically, we firstly rewrite the energy harvesting constraint in terms of $\alpha_{n}$ as

$$
\eta_{E}\left\|\boldsymbol{H}_{E} \boldsymbol{v}_{I}\right\|^{2}=C_{n}+\alpha_{n} D_{n}+\alpha_{n}^{*} D_{n}^{*}, \quad \forall n \in \mathcal{N},
$$

where

$C_{n}=\eta_{E}\left\|\left(\boldsymbol{H}_{d, E}+\sum_{m=1, m \neq n}^{N_{R}} \alpha_{m} \boldsymbol{h}_{r, E}^{m} \boldsymbol{g}_{m}^{H}\right) \boldsymbol{v}_{I}\right\|^{2}+\eta_{E}\left\|\boldsymbol{h}_{r, E}^{n}\right\|^{2}\left|\boldsymbol{g}_{n}^{H} \boldsymbol{v}_{I}\right|^{2}$,

$D_{n}=\eta_{E} \boldsymbol{v}_{I}^{H}\left(\boldsymbol{H}_{d, E}+\sum_{m=1, m \neq n}^{N_{R}} \alpha_{m} \boldsymbol{h}_{r, E}^{m} \boldsymbol{g}_{m}^{H}\right)^{H} \boldsymbol{h}_{r, E}^{n} \boldsymbol{g}_{n}^{H} \boldsymbol{v}_{I}$. 
Let's consider $D_{n}=\left|D_{n}\right| e^{j \theta_{n}}$, the optimal $\alpha_{n}$ to problem (5) for any given $\boldsymbol{v}_{I}$ and $\left\{\alpha_{m}\right\}_{m=1, m \neq n}^{N_{R}}$ is expressed as

$\alpha_{n}=\underset{\alpha}{\arg \max } \cos \left(\operatorname{Pha}(\alpha)+\psi_{n}\right)$,

s.t. $\operatorname{Pha}(\alpha) \in \mathcal{P}, \cos \left(\operatorname{Pha}(\alpha)+\theta_{n}\right) \geq E_{0}-C_{n} /\left(2\left|D_{n}\right|\right)$.

In a nutshell, for both two distinct cases of $E_{0}$, the objective value of problem (5) is non-decreasing over the iterations, where the individual IRS reflection coefficients $\alpha_{n}$ 's are successively and uniquely updated according to (20) or (23) for any given $\boldsymbol{v}_{I}$, and is also upper-bounded by a finite value corresponding to the maximum power transmission [24]. Moreover, by recalling Proposition 2, the unique optimal solution of the subproblem w.r.t $\boldsymbol{v}_{I}$ in the case of $E_{0} \in\left[E_{0}^{\mathrm{mi}}, E_{0}^{\max }\right]$ is also available. Therefore, similarly to that in Section III-A, we can conclude that regardless of the value range of the energy threshold $E_{0}$, the proposed lowcomplexity alternating optimization algorithm is guaranteed to converge to a locally optimal solution of problem (5) [29]. The computational complexity of this alternating optimization algorithm is shown to be $\mathcal{O}\left(\left(N_{R} N_{U} N_{S}+L\right) I_{A}\right)$, where $I_{A}$ denotes the number of iterations.

\section{MIME SCENARIO}

In this section, we consider the MIME scenario, where multiple IDs and multiple ERs operate in the same timefrequency resource and both are aided by the IRS. Similarly to the SISE scenario, we firstly study the feasibility and tightness analysis of problem (P1). Then an equivalent power minimization reformulation of problem (P1) is introduced. To tackle the non-convexity of this equivalent problem, an alternating optimization algorithm is accordingly proposed. Furthermore, we develop a low-complexity two-stage scheme for further reducing the iteration complexity.

\section{A. Feasibility and Tightness Analysis}

In the general MIME scenario, the feasibility problem of problem (P1) is formulated as

$$
\begin{aligned}
\max _{\left\{\boldsymbol{v}_{i_{I}}\right\}, \boldsymbol{\Phi}} & \sum_{i_{E} \in \mathcal{K}_{E}} \sum_{i_{I} \in \mathcal{K}_{I}} \eta_{i_{E}}\left\|\boldsymbol{H}_{i_{E}} \boldsymbol{v}_{i_{I}}\right\|^{2}=\sum_{i_{I} \in \mathcal{K}_{I}} \boldsymbol{v}_{i_{I}}^{H} \overline{\boldsymbol{H}}_{E} \boldsymbol{v}_{i_{I}} \\
\text { s.t. } & \sum_{i_{I} \in \mathcal{K}_{I}}\left\|\boldsymbol{v}_{i_{I}}\right\|^{2} \leq P_{0}, \boldsymbol{\Phi}=\operatorname{diag}\left[\alpha_{1}, \cdots, \alpha_{N_{R}}\right], \\
& \operatorname{Pha}\left(\alpha_{n}\right) \in \mathcal{P}, \quad \forall n \in \mathcal{N} .
\end{aligned}
$$

where $\overline{\boldsymbol{H}}_{E}=\sum_{i_{E} \in \mathcal{K}_{E}} \eta_{i_{E}} \boldsymbol{H}_{i_{E}}^{H} \boldsymbol{H}_{i_{E}}$. Similarly to problem (6), the optimal $\left\{\boldsymbol{v}_{i_{I}}\right\}$ to problem (24) for any given $\boldsymbol{\Phi}$ is readily obtained as $\boldsymbol{v}_{i_{I}}^{\star}=\sqrt{p_{i_{I}}} \boldsymbol{\mu}_{\max }\left(\overline{\boldsymbol{H}}_{E}\right), \forall i_{I} \in \mathcal{K}_{I}$, where $p_{i_{I}}$ 's are arbitrary nonnegative numbers satisfying $\sum_{i_{I} \in \mathcal{K}_{I}} p_{i_{I}}=P_{0}$, indicating that all information beams should be aligned to the same direction $\boldsymbol{v}_{E}$ for maximizing the harvested energy. Furthermore, substituting the resultant optimal $\boldsymbol{v}_{i_{I}}$ 's into problem (24) yields

$$
\begin{aligned}
\max _{\left\{\boldsymbol{v}_{i_{I}}\right\}, \boldsymbol{\Phi}} & P_{0} \lambda_{\max }\left(\overline{\boldsymbol{H}}_{E}\right) \\
\text { s.t. } & \boldsymbol{\Phi}=\operatorname{diag}\left[\alpha_{1}, \cdots, \alpha_{N_{R}}\right], \operatorname{Pha}\left(\alpha_{n}\right) \in \mathcal{P}, \forall n \in \mathcal{N} .
\end{aligned}
$$

Obviously, problem (25) has a similar form to problem (7), and thereby can also be rewritten in an explicit form of

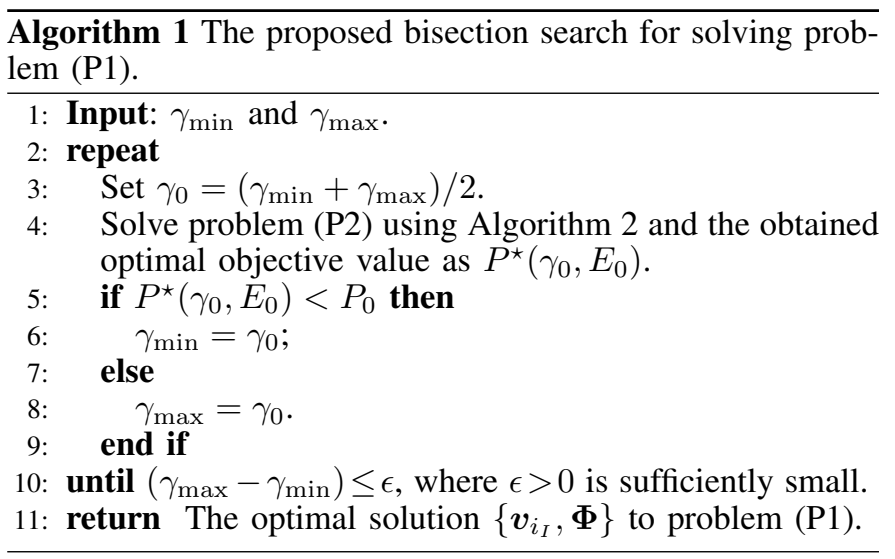

Algorithm 2 The proposed alternating optimization algorithm for solving problem (P2).

1: Input: Initial $\left\{\alpha_{m}^{(0)}\right\}$ and iteration index $I_{T}=0$.

2: repeat

3: Given $\left\{\alpha_{m}^{\left(I_{T}\right)}\right\}$, solve the SDP problem (28) to obtain the optimal $\boldsymbol{v}_{i_{I}}^{\left(I_{T}+1\right)}, \forall i_{I} \in \mathcal{K}_{I}$.

4: $\quad$ Set $\overline{\boldsymbol{v}}_{i_{I}}=\boldsymbol{v}_{i_{I}}^{\left(I_{T}+1\right)}, \forall i_{I} \in \mathcal{K}_{I}$.

5: $\quad$ repeat

6: $\quad$ for $n=1 \rightarrow N_{R}$ do

7: $\quad$ Given $\overline{\boldsymbol{v}}_{i_{I}}$ 's and $\left\{\alpha_{m}^{\left(I_{T}\right)}\right\}_{m=1, m \neq n}^{N_{R}}$, calculate $\alpha_{n}$ according to (33).

Update $\alpha_{n}^{\left(I_{T}\right)}=\alpha_{n}$.

end for

until The relative increment in the objective value $\gamma_{\text {mar }}$ of problem (33) is sufficiently small.

11: $\quad$ return $\alpha_{n}^{\left(I_{T}+1\right)}=\alpha_{n}^{\left(I_{T}\right)}, \forall n=1, \cdots, N_{R}$.

12: $\quad$ Set $I_{T}=I_{T}+1$.

13: Given $\boldsymbol{v}_{i_{I}}^{\left(I_{T}+1\right)}$, s and $\left\{\alpha_{n}^{\left(I_{T}+1\right)}\right\}$, calculate the optimal objective value $P^{\left(I_{T}\right)}\left(\gamma_{0}, E_{0}\right)$ of problem $(\mathrm{P} 2)$.

14: until $\left|P^{\left(I_{T}\right)}\left(\gamma_{0}, E_{0}\right)-P^{\left(I_{T}-1\right)}\left(\gamma_{0}, E_{0}\right)\right| \leq \epsilon$, where $\epsilon$ is a sufficiently small positive number.

15: return $P^{\star}\left(\gamma_{0}, E_{0}\right)=P^{\left(I_{T}\right)}\left(\gamma_{0}, E_{0}\right)$.

$\alpha_{n}$ like problem (8). Naturally, for a small-scale system, an exhaustive search is expected to find the globally optimal $\boldsymbol{\Phi}$, while for a medium-to-large scale system, we intend to apply the alternating optimization algorithm proposed for solving problem (7) to obtain a locally optimal $\boldsymbol{\Phi}$ of problem (24). Additionally, for the MIME scenario, we find that the tightness analysis of the energy harvesting constraint relies on solving the minimum SINR maximization problem in the IRS-aided multiuser MISO system, which is still intractable due to its strongly coupled variables. As such, an accurate crucial energy threshold $E_{0}^{\mathrm{mi}}$ is hard to obtain. More importantly, as will be illustrated later, the proposed alternating optimization algorithm for the MIME scenario is directly applicable regardless of the tightness of the energy harvesting constraint. Therefore, we omit this intractable and trivial tightness analysis here for simplicity.

\section{B. Equivalent Reformulation}

To address the nonconvex problem (P1), we firstly present the following proposition.

Proposition 3. Consider the following power minimization problem $^{4}$ :

${ }^{4}$ Note that for the arbitrary $\gamma_{0}$ and $E_{0}$, problem (26) is always feasible by scaling up the power of the BS transmit beamforming vectors $\boldsymbol{v}_{i_{I}}$ 's. 


$$
\begin{aligned}
& (\mathrm{P} 2): \min _{\boldsymbol{v}_{i_{I}}, \boldsymbol{\Phi}} \sum_{i_{I} \in \mathcal{K}_{I}}\left\|\boldsymbol{v}_{i_{I}}\right\|^{2} \\
& \text { s.t. } \sum_{i_{E} \in \mathcal{K}_{E}} \sum_{i_{I} \in \mathcal{K}_{I}} \eta_{i_{E}}\left\|\boldsymbol{H}_{i_{E}} \boldsymbol{v}_{i_{I}}\right\|^{2} \geq E_{0}, \\
& \frac{\left|\boldsymbol{h}_{i_{I}}^{H} \boldsymbol{v}_{i_{I}}\right|^{2}}{\sum_{j_{I} \neq i_{I}}\left|\boldsymbol{h}_{i_{I}}^{H} \boldsymbol{v}_{j_{I}}\right|^{2}+\sigma_{i_{I}}^{2}} \geq \gamma_{0}, \quad \forall i_{I} \in \mathcal{K}_{I}, \\
& \mathbf{\Phi}=\operatorname{diag}\left[\alpha_{1}, \cdots, \alpha_{N_{R}}\right], \operatorname{Pha}\left(\alpha_{n}\right) \in \mathcal{P}, \forall n \in \mathcal{N} .
\end{aligned}
$$

Let's define the optimal objective values of problems $(P 1)$ and (P2) as $\gamma^{\star}\left(P_{0}, E_{0}\right)$ and $P^{\star}\left(\gamma_{0}, E_{0}\right)$, respectively. Then we have $P_{0}=P^{\star}\left(\gamma^{\star}\left(P_{0}, E_{0}\right), E_{0}\right)$ which implies that problem $(P 2)$ is an inverse counterpart of problem (P1). Moreover, for any given $E_{0}$, it yields

$$
\tilde{\gamma}_{0}>\gamma_{0} \Rightarrow P^{\star}\left(\tilde{\gamma}_{0}, E_{0}\right) \geq P^{\star}\left(\gamma_{0}, E_{0}\right) .
$$

Proof. Please see Appendix C.

It is worth noting that although this inversion property has been well studied for the minimum SINR maximization problem of traditional multiuser MISO systems, its extension to our work is challenging, since an extra nonconvex energy harvesting constraint is considered. Armed with the inversion property in Proposition 3, it can be inferred that the optimal solution to problem (P1) can be efficiently found by iteratively solving problem (P2) for different $\gamma_{0}$ 's until its objective value satisfies $P^{\star}\left(\gamma_{0}, E_{0}\right)=P_{0}$. Moreover, according to the monotonically non-decreasing property of $P^{\star}\left(\gamma_{0}, E_{0}\right)$ in $\gamma_{0}$, the optimal $\gamma_{0}=\gamma^{\star}\left(P_{0}, E_{0}\right)$ can be uniquely determined by a simple bisection search. For simplicity, we set the lower bound $\gamma_{\min }$ of $\gamma_{0}$ to zero, while its upper bound $\gamma_{\max }$ is calculated by with the interference power neglected. The specific algorithm procedure is further summarized in Algorithm 1.

Although the inverse problem (P2) with the quadratic objective function is easier to handle than the original problem (P1), it is still difficult to directly address due to the involved individual nonconvex SINR constraints. In the sequel, from the perspective of decoupling optimization variables, we consider decomposing problem (P2) into two subproblems w.r.t. $\boldsymbol{v}_{i_{I}}$ 's and $\boldsymbol{\Phi}$, respectively. Moreover, an alternating optimization algorithm is proposed to derive a high-quality suboptimal solution of problem (P2).

\section{Alternating Optimization}

In this subsection, assuming an arbitrary feasible energy threshold $E_{0}$, we aim to solve problem (P2) in an iterative manner. Firstly, for any given $\boldsymbol{\Phi}$, we consider using the SDR technique to tackle the non-convexity of problem (P1) w.r.t $\boldsymbol{v}_{i_{I}}$ 's. To be specific, let's define positive semidefinite matrices $\boldsymbol{V}_{i_{I}}=\boldsymbol{v}_{i_{I}} \boldsymbol{v}_{i_{I}}^{H}, \forall i_{I} \in \mathcal{K}_{I}$. Then, by temporarily neglecting the implicit rank-1 constraints on $\boldsymbol{V}_{i_{I}}$ 's, problem (P2) is relaxed to the following SDP problem

$$
\begin{aligned}
& \min _{\left\{\boldsymbol{V}_{i_{I}}\right\} \succeq \mathbf{0}} \sum_{i_{I} \in \mathcal{K}_{I}} \operatorname{tr}\left(\boldsymbol{V}_{i_{I}}\right) \\
& \text { s.t. } \sum_{i_{E} \in \mathcal{K}_{E}} \sum_{i_{I} \in \mathcal{K}_{I}} \eta_{i_{E}} \operatorname{tr}\left(\boldsymbol{H}_{i_{E}}^{H} \boldsymbol{H}_{i_{E}} \boldsymbol{V}_{i_{I}}\right) \geq E_{0}, \\
& \operatorname{tr}\left(\boldsymbol{h}_{i_{I}} \boldsymbol{h}_{i_{I}}^{H} \boldsymbol{V}_{i_{I}}-\sum_{j_{I} \neq i_{I}} \gamma_{0} \boldsymbol{h}_{i_{I}} \boldsymbol{h}_{i_{I}}^{H} \boldsymbol{V}_{j_{I}}\right)-\gamma_{0} \sigma_{i_{I}}^{2} \geq 0, \quad \forall i_{I} \in \mathcal{K}_{I},
\end{aligned}
$$

It is further validated in the following proposition that problem (P2) can be relaxed to problem (28) without loss of optimality.

Proposition 4. The optimal solution of problem (28) satisfies $\operatorname{rank}\left(\boldsymbol{V}_{i_{I}}^{\star}\right)=1, \forall i_{I} \in \mathcal{K}_{I}$ with probability one.

\section{Proof. Please see Appendix D.}

According to Proposition 4, the optimal $\boldsymbol{v}_{i_{I}}$ 's to problem (P2) with any given $\boldsymbol{\Phi}$ can be found by solving the convex SDP problem (28) instead. Nevertheless, since the objective of transmit power minimization in problem (P2) is not a function of $\boldsymbol{\Phi}$, the optimization of $\boldsymbol{\Phi}$ with any given $\boldsymbol{v}_{i_{I}}$ 's in essence belongs to a feasibility problem. For the sake of finding the effective IRS reflection coefficients to guarantee the convergence of the proposed alternating optimization algorithm, we next consider creating an explicit objective for the optimization of $\boldsymbol{\Phi}$. Specifically, motivated by the fact that there is at least one constraint in $(26 b) \sim(26 c)$ being active at the optimal solution of problem $(\mathrm{P} 2)$, we propose maximizing the minimum margin associated with individual SINRs of all IDs and the total harvested energy at all ERs for the optimization of IRS reflection coefficients. Note that this design also enables future reduction of the total transmit power in the following optimization of the BS transmit beamforming (i.e. by simply scaling down the BS transmit beamforming power).

To this end, with the aid of the normalization technique, we firstly transfer both the output SINR of each ID and the total harvested energy of all ERs into the following dimensionless functions to make them commensurable.

$$
\begin{aligned}
& \operatorname{SINR}_{i_{I}, \text { nor }}=\frac{\left|\boldsymbol{h}_{i_{I}}^{H} \boldsymbol{v}_{i_{I}}\right|^{2}}{\gamma_{0}\left(\sum_{j_{I} \neq i_{I}}\left|\boldsymbol{h}_{i_{I}}^{H} \boldsymbol{v}_{j_{I}}\right|^{2}+\sigma_{i_{I}}^{2}\right)}, \\
& E_{\text {nor }}=\frac{\sum_{i_{E} \in \mathcal{K}_{E}} \sum_{i_{I} \in \mathcal{K}_{I}} \eta_{i_{E}} \operatorname{tr}\left(\boldsymbol{H}_{i_{E}}^{H} \boldsymbol{H}_{i_{E}} \boldsymbol{V}_{i_{I}}\right)}{E_{0}}, \quad \forall i_{I} \in \mathcal{K}_{I} .
\end{aligned}
$$

Based on (29), we then define the total harvested energy margin of all ERs as $E_{\text {mar }}=E_{\text {nor }}-1$, and the SINR margin of each ID as

$$
\begin{aligned}
\operatorname{SINR}_{i_{I}, \text { mar }} & =\operatorname{SINR}_{i_{I}, \text { nor }}-1 \\
& \leq \frac{\left|\boldsymbol{h}_{i_{I}}^{H} \boldsymbol{v}_{i_{I}}\right|^{2}-\gamma_{0}\left(\sum_{j_{I} \neq i_{I}}\left|\boldsymbol{h}_{i_{I}}^{H} \boldsymbol{v}_{j_{I}}\right|^{2}+\sigma_{i_{I}}^{2}\right)}{\gamma_{0} \sigma_{i_{I}}^{2}} \\
& =\overline{\operatorname{SINR}}_{i_{I}, \text { mar }}, \quad \forall i_{I} \in \mathcal{K}_{I},
\end{aligned}
$$

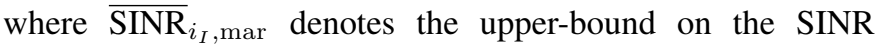
margin of ID $i_{I}$, and is obtained by neglecting the positive term $\sum_{j_{I} \neq i_{I}}\left|\boldsymbol{h}_{i_{I}}^{H} \boldsymbol{v}_{j_{I}}\right|^{2}$ in the denominator. This upper-bound also leads to the potential improvement of the output SINR of each ID, since it has the same numerator as the original SINR margin and a positive constant denominator. Motivated by this fact, for finding an effective $\boldsymbol{\Phi}$ to problem (P2) with any given $\boldsymbol{v}_{i_{I}}$ 's, we further formulate the maximization of the minimum margin over the individual SINRs of all IDs and the total harvested energy as

$$
\begin{aligned}
& \max _{\mathbf{\Phi}} \min _{i_{I} \in \mathcal{K}_{I} \cup 0}\left\{\mathrm{SINR}_{i_{I}, \text { mar }}, E_{\text {mar }}\right\}, \\
& \text { s.t. } \quad E_{\text {mar }} \geq 0, \mathrm{SINR}_{i_{I}, \text { mar }} \geq 0, \forall i_{I} \in \mathcal{K}_{I},
\end{aligned}
$$


where $i_{I}=0$ denotes the index of the argument $E_{\text {mar }}$. Further, by introducing an auxiliary variable $\gamma_{\text {mar }}$, problem (31) can be equivalently transformed into

$$
\begin{array}{ll}
\max _{\mathbf{\Phi}} & \gamma_{\text {mar }}, \\
\text { s.t. } & \gamma_{\text {mar }} \geq 0, E_{\text {mar }} \geq \gamma_{\text {mar }}, \quad \operatorname{SINR}_{i_{I}, \text { mar }} \geq \gamma_{\text {mar }}, \forall i_{I} \in \mathcal{K}_{I} .
\end{array}
$$

Recall Proposition 1, problem (32) can still be optimally solved by the branch-and-bound algorithm by jointly applying

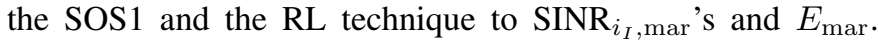
Nonetheless, due to the increased number of 0-1 quadratic constraints associated with multiple IDs, problem (32) is generally more time-consuming to address than problem (5) with any given $\boldsymbol{v}_{I}$, especially for a large $N_{R}$ (i.e. $N_{R} \geq 36$ ). As a result, we consider again the element-by-element iterative algorithm proposed in Section III-C. To be specific, the optimal $\alpha_{n}$ to problem (32) with any given $\left\{\alpha_{m}\right\}_{m=1, m \neq n}^{N_{R}}$ is readily determined by a one-dimensional search over $\mathcal{P}$, which is formulated as

$$
\begin{aligned}
\alpha_{n}^{\star}=\underset{\operatorname{Pha}(\alpha) \in \mathcal{P}}{\arg \max } \min _{i_{I} \in \mathcal{K}_{I} \cup 0}\left\{E_{\operatorname{mar}}\left(\left\{\alpha_{m}\right\}_{m=1, m \neq n}^{N_{R}}, \alpha\right),\right. \\
\left.\operatorname{SINR}_{i_{I}, \operatorname{mar}}\left(\left\{\alpha_{m}\right\}_{m=1, m \neq n}^{N_{R}}, \alpha\right)\right\}, \forall n \in \mathcal{N} .
\end{aligned}
$$

By iteratively updating individual IRS reflection coefficients $\alpha_{n}$ 's according to (32) until the relative increment of $\gamma_{\text {mar }}$ becomes sufficiently small, we can obtain a sequence of nondecreasing minimum margins, implying a certain reduction of the BS transmit power in the subsequent optimization of BS transmit beamforming vectors $\boldsymbol{v}_{i_{I}}$ 's.

Overall, the proposed alternating optimization algorithm for solving problem (P2) consists of optimizing the BS transmit beamforming vectors $\boldsymbol{v}_{i_{I}}$ 's by using the SDR technique and the IRS reflection matrix $\boldsymbol{\Phi}$ by introducing an explicit optimization objective. Note that the SDR is proved to be tight in Proposition 4, and thus the unique optimal $\boldsymbol{v}_{i_{I}}$ 's are available. In contrast, the design of $\boldsymbol{\Phi}$ belongs to a feasibility problem whose optimization objective is inexplicit and nonunique, which thus makes the convergence result in the SISE scenario not directly applicable. In fact, since the continuous objective function of problem (P2) is minimized at each iteration, we readily infer that this alternating optimization algorithm yields non-increasing objective values of problem (P2) over the iterations, which is also lower-bounded due to the closed feasible set. However, as mentioned above, the iterates generated by this algorithm cannot be guaranteed to converge to the B-stationary solution of problem (P2).

As a result, this element-by-element iterative algorithm is able to converge to a locally optimal solution of problem (32). Inspired by the design philosophy of the IRS reflection coefficients and the uniquely optimal solution of the subproblem w.r.t. $\boldsymbol{v}_{i_{I}}$ 's, we can infer that the proposed alternating optimization algorithm is guaranteed to converge to a highquality suboptimal solution of problem (P2).

Additionally, the computational complexity of the proposed alternating optimization algorithm mainly lies in iteratively solving a pair of subproblems w.r.t $\boldsymbol{v}_{i_{I}}$ 's and $\boldsymbol{\Phi}$. On the one hand, the subproblem (28) w.r.t $\boldsymbol{v}_{i_{I}}$ 's is a standard convex SDP problem with $K_{I}$ LMI constraints of size $N_{S}$ and $K_{I}+1$ linear constraints, whose complexity is on the order of $\mathcal{O}_{S_{1}}\left(\left(K_{I} N_{S}+K_{I}+1\right)^{0.5} K_{I}^{3} N_{S}^{6}\right) \quad$ [31]. On the other hand, the adopted element-by-element iterative algorithm for the subproblem (32) w.r.t $\boldsymbol{\Phi}$ has the complexity of $\mathcal{O}_{S_{2}}\left(I_{A} L N_{R}^{2} N_{S}\left(K_{I}+N_{E}\right)\right)$, where $I_{A}$ denotes the number of iterations. Hence, the total complexity of solving problem (P2) is given by $I_{\text {out }}\left(I_{T}\left(\mathcal{O}_{S_{1}}+\mathcal{O}_{S_{2}}\right)\right)$, where $I_{T}$ denotes the number of iterations between the subproblems (28) and (32). We will demonstrate in numerical simulations that the proposed alternating optimization algorithm for solving problem (P2) works well in practice and converges to the maximum after about 10 iterations.

\section{Two-Stage Scheme}

In this subsection, to further reduce the computational complexity of the above proposed alternating optimization algorithm, we conceive a two-stage scheme for separately optimizing the BS transmit beamforming vectors $\boldsymbol{v}_{i_{I}}$ 's and the IRS reflection matrix $\boldsymbol{\Phi}$. Specifically, in the first stage, we aim to optimize the IRS reflection matrix $\boldsymbol{\Phi}$ so that the weighted total channel power gain is maximized, which can be formulated as

$$
\begin{aligned}
\max _{\boldsymbol{\Phi}} & \sum_{i_{I} \in \mathcal{K}_{I}} w_{i_{I}}\left\|\boldsymbol{h}_{i_{I}}\right\|^{2}+\sum_{i_{E} \in \mathcal{K}_{E}} w_{i_{E}}\left\|\boldsymbol{H}_{i_{E}}\right\|_{F}^{2}, \\
\text { s.t. } & \boldsymbol{\Phi}=\operatorname{diag}\left[\alpha_{1}, \cdots, \alpha_{N_{R}}\right], \operatorname{Pha}\left(\alpha_{n}\right) \in \mathcal{P}, \forall n \in \mathcal{N} .
\end{aligned}
$$

where the weighting factors $w_{i_{I}}=\frac{1}{\gamma_{0} \sigma_{i_{I}}^{2}}, \forall i_{I} \in \mathcal{K}_{I}$ and $w_{i_{E}}=\frac{\eta_{i_{E}}}{E_{0}} \forall i_{E} \in \mathcal{K}_{E}$ are chosen, considering the proportional fairness among the channel gains of all IDs and ERs implied by the first two constraints in problem (P2). Note that this IRS reflection matrix design aims to construct the wireless propagation environment with high information and energy beamforming gains by adjusting the phases of different channels from the BS to all IDs and ERs. Since problem (34) has a similar structure to problem (12), the proposed low-complexity alternating optimization algorithm in Section III-C can still be applied to obtain a monotone non-decreasing sequence of the weighted total channel gains, which ultimately converges to a locally optimal solution of problem (34).

In the second stage, we aim to design the BS transmit beamforming vectors $\boldsymbol{v}_{i_{I}}$ 's by solving problem (P1) with $\boldsymbol{\Phi}$ obtained by solving problem (34). In contrast to the above passive IRS reflection coefficients design, this active BS transmit beamforming design aims to enhance the minimum output SINR among all IDs as much as possible by utilizing both the full power transmission and the effective inter-user interference suppression. Moreover, by recalling Proposition 3 and Proposition 4, the corresponding globally optimal $\boldsymbol{v}_{i_{I}}$ 's can also be derived from Algorithm 1.

The overall complexity of the two-stage scheme is on the order of $\mathcal{O}\left\{I_{A} L N_{R}^{2} N_{S}\left(K_{I}+N_{E}\right)+I_{B} K_{I} N_{S}+K_{I}+\right.$ 1) $\left.{ }^{0.5} K_{I}^{3} N_{S}^{6}\right\}$, where $I_{A}$ and $I_{B}$ denote the number of iterations required for finding the optimal $\boldsymbol{\Phi}$ to problem (34) and the optimal $\boldsymbol{v}_{i_{I}}$ 's using bisection search, respectively. Compared to the above proposed alternating optimization algorithm, this two-stage scheme generally has much lower computational complexity since only a single iteration is performed between 
the optimization of $\boldsymbol{\Phi}$ and that of $\boldsymbol{v}_{i_{I}}$ 's. As will be illustrated in numerical simulations, the proposed two-stage scheme performs comparably to the alternating optimization algorithm in the high SNR regime.

\section{Simulations}

This section presents numerical simulation results for characterizing the performance of our proposed algorithms in terms of the output SINR performance. As illustrated in Fig. 2, we consider a three-dimensional coordinate system, where a uniform linear array (ULA) with half-wavelength spaced elements along the $x$-axis is mounted at both the BS and each ER, while the IRS installs a uniform planar array (UPA) with elements spaced along the $x$-axis and $y$-axis every quarter-wavelength, respectively. The locations of the reference antenna at the BS and IRS are set as $(0,0,0) \mathrm{m}$ and $\left(0, d_{\mathrm{R}}, 1\right) \mathrm{m}$, respectively. In particular, we consider an isotropic radiation pattern at the BS and thus its antenna gain is $0 \mathrm{dBi}$. Nevertheless, since the IRS reflects signal only through the front half-sphere, it is assumed to have $3 \mathrm{dBi}$ antenna gain [6]. For the sake of improving energy harvesting efficiency, we also consider the horizontal projection of the IRS's location (i.e. $\left(0, d_{\mathrm{R}}, 0\right)$ $\mathrm{m})$ as a EH hotspot and assume that all ERs are randomly located within a circular cluster centered at this hotspot with a radius of $r_{\mathrm{E}} \mathrm{m}$. Similarly, all single-antenna IDs are uniformly distributed within a circular cluster of radius $r_{\mathrm{I}} \mathrm{m}$, centered at a location $\left(0,0, d_{\mathrm{D}}\right) \mathrm{m}$. The large-scale path loss model is given by $\operatorname{PL}(d)=\beta_{0}\left(d / d_{0}\right)^{-\alpha}$, where $\beta_{0}=-30 \mathrm{~dB}$ denotes the signal power attenuation at a reference distance $d_{0}=1 \mathrm{~m}$ and $\alpha>0$ is path loss factor, respectively. Considering that the IRS is usually deployed to assist SWIPT from the BS to IDs and ERs suffering from severe signal power attenuation, we set the path loss factor of the BS-ID (BS-ER) channel to $\alpha_{\mathrm{BU}}=3.6$, while those of the BS-IRS and IRS-ID (IRS-ER) channels to $\alpha_{\mathrm{BI}}=2.1$ and $\alpha_{\mathrm{IU}}=2.3$, respectively.

Furthermore, the small-scale fading of all wireless channels is modeled by Rician distribution $\boldsymbol{H}=\sqrt{\frac{\alpha K_{\mathrm{R}}}{1+K_{\mathrm{R}}}} \boldsymbol{H}^{\mathrm{LoS}}+$ $\sqrt{\frac{\alpha}{1+K_{\mathrm{R}}}} \boldsymbol{H}^{\mathrm{NLoS}}$, where $K_{\mathrm{R}}$ denotes the Rician $K$-factor. $\boldsymbol{H}^{\mathrm{LoS}}$ and $\boldsymbol{H}^{\mathrm{NLoS}}$ are the LoS and NLoS components of channel $\boldsymbol{H}$, respectively. The elements of the NLoS component $\boldsymbol{H}^{\mathrm{NLoS}}$ $\left(G^{\mathrm{NLoS}}\right)$ are assumed to be Rayleigh distributed with zero mean and unit variance, while the LoS component $G^{\text {NLoS }}$ $\left(G^{\mathrm{NLoS}}\right)$ is usually characterized by a product of the array steering vectors at both communication ends, where the corresponding elevation and azimuth angles are assumed to be uniformly distributed over the intervals $\theta \in\left[-\frac{\pi}{2}, \frac{\pi}{2}\right]$ and $\psi \in[0,2 \pi)$, respectively [8]. Unless otherwise stated, the BS-ID (BS-ER), BS-IRS and IRS-ID (IRS-ER) channels are modeled using Rician factors $K_{\mathrm{R}}^{\mathrm{BU}}=0, K_{\mathrm{R}}^{\mathrm{BI}}=10 \mathrm{~dB}$ and $K_{\mathrm{R}}^{\mathrm{IU}}=\infty$, respectively. Moreover, we set the maximum transmit power at the BS to $P_{0}=25 \mathrm{dBm}$. The noise power spectral density of $-169 \mathrm{dBm} / \mathrm{Hz}$ and the system bandwidth of $10 \mathrm{MHz}$ are assumed, and the energy conversion efficiency is chosen as $\eta_{i_{E}}=0.6, \forall i_{E} \in \mathcal{K}_{E}$. Furthermore, the relative convergence threshold of all proposed alternating optimization (Alt-Opt) algorithms is determined as $10^{-4}$. The numbers of antennas at the BS and each ER (i.e. $N_{S}$ and $N_{U}$ ) are specified

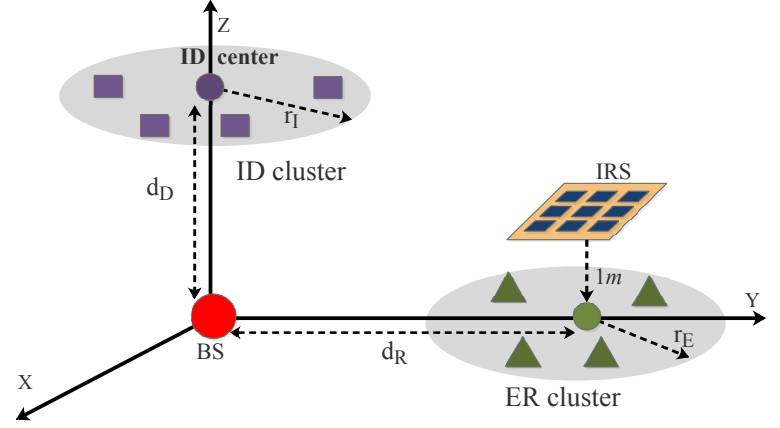

Fig. 2. Simulation setup of the general IRS-aided SWIPT system.

later. Note that all simulation points are obtained by averaging over 100 independent channel realizations.

\section{A. Initialization and Comparison}

Recall that we have proposed various kinds of lowcomplexity alternating optimization (Alt-Opt) algorithms for optimizing the SNR performance in the SISE scenario and the minimum SINR performance among all IDs in the MIME scenario, respectively. Generally, the initial IRS reflection coefficients play an important role in ensuring the effectiveness of the proposed Alt-Opt algorithms. As such, we mainly consider three different initialization methods for the proposed algorithms, as elaborated below: 1) Total harvested energy maximization: The corresponding optimal IRS reflection coefficients are obtained by solving problem (8) in an iterative manner. With this initialization, the feasibility of problem (P1) can be guaranteed; 2) Total BS-ID channel gain maximization: The optimal design of the IRS reflection coefficients aims to maximize the total BS-ID channel gain of $\sum_{i_{I} \in \mathcal{K}_{I}}\left\|\boldsymbol{h}_{i_{I}}^{H}\right\|^{2}$, and can also be realized by an iterative algorithm. Notice that this initialization method performs well when the energy harvesting constraint is inactive (as explained later); 3) Total BS-ER channel gain maximization: Like in case 2), we derive the optimal IRS reflection coefficients by maximizing the total BS-ER channel gain $\sum_{i_{E} \in \mathcal{K}_{E}}\left\|\boldsymbol{H}_{i_{E}}\right\|^{2}$.

Furthermore, in order to comprehensively evaluate the performance of all our proposed algorithms in both SISE and MIME scenarios, we also consider the following three benchmark schemes for comparisons: 1) Upper-bound scheme: We solve problem (P1) under the assumption of continuous IRS phase shifts, i.e. $b \rightarrow \infty$ by jointly using semidefinite relaxation (SDR) technique and Gaussian randomization proposed in [6]; 2) Quantization-based scheme (QBS): In this scheme, we individually quantize the continuous IRS phase shifts obtained in case 1) into the nearest values in the predefined set of discrete phase shifts; 3)Without IRS: Assuming $\boldsymbol{\Phi}=\mathbf{0}$, the globally optimal BS transmit beamforming vectors to problem (P1) are then derived.

\section{B. SISE scenario}

In this subsection, we focus on the SISE scenario and study the maximum achievable SNR of the ID, while guaranteeing the energy harvesting requirement at the ER. Unless specified otherwise, we set $N_{S}=4, N_{R}=32, N_{U}=3, d_{\mathrm{R}}=10$ and $d_{\mathrm{I}}=60$. The locations of a single ID and a single ER are denoted by $(0,0,60) \mathrm{m}$ and $(0,10,0) \mathrm{m}$, respectively. Furthermore, $b=2$ quantization bits are used for characterizing the set of IRS discrete phase shifts. 


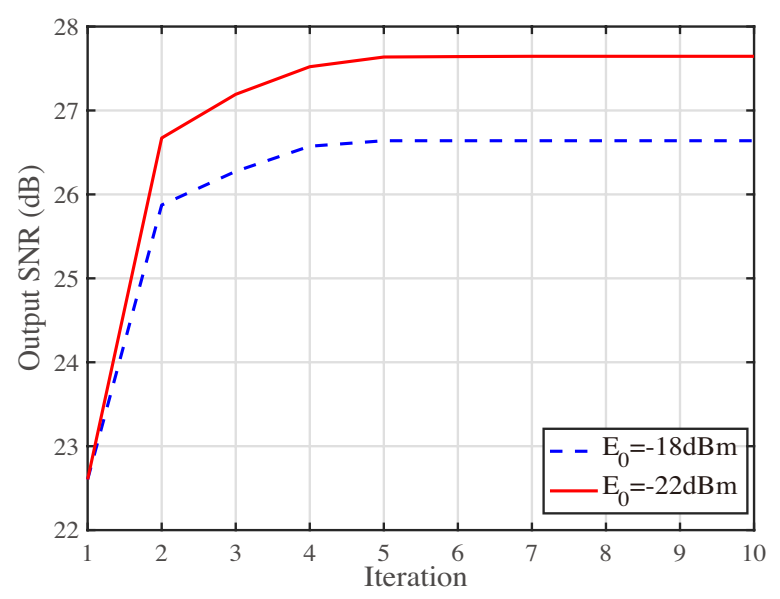

(a) Convergence behavior under both inactive and active energy thresholds

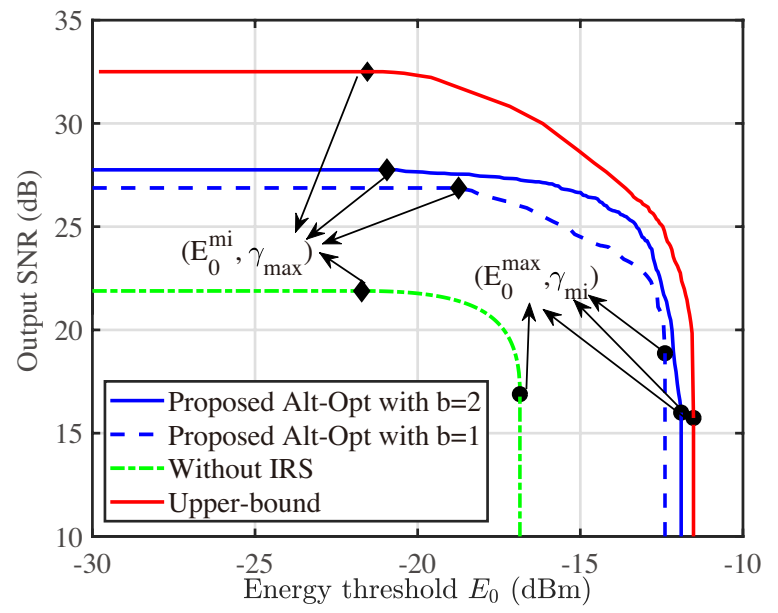

(b) Achievable SNR-energy regions

Fig. 3. Performance of the proposed Alt-Opt algorithms in the SISE scenario.

Fig. 3 (a) firstly shows the convergence behavior (under one channel realization) of the proposed low-complexity AltOpt algorithm in Section III-C for solving problem (5) under both the inactive and active energy harvesting constraints, corresponding to $E_{0}=-22 \mathrm{dBm}$ and $E_{0}=-18 \mathrm{dBm}$, respectively. For each case, it can be seen that the Alt-Opt algorithm converges to the maximum achievable SNR within 10 iterations. Moreover, much of the SNR improvement occurs in the first a few iterations, which indicates that the proposed Alt-Opt algorithm has a low complexity. Obviously, the output SNR achieved under $E_{0}=-22 \mathrm{dBm}$ is higher than that under $E_{0}=-18 \mathrm{dBm}$ due to the enlarged size of the feasible solution region.

In Fig. 3 (b), the SNR-energy regions characterized by the proposed Alt-Opt algorithm in Section III-C and benchmarks are presented, where both $b=1$ and $b=2$ are considered for phase quantization. Other parameters are the same as those in Fig. 3 (a). It is firstly observed that the upper-bound scheme using continuous phase shifts realize the largest rateenergy region. With the aid of the IRS, the proposed Alt-Opt algorithms with $b=1$ and $b=2$ both characterize a larger rate-energy region than the benchmark scheme without IRS. Furthermore, for the same energy threshold $E_{0}$, the Alt-Opt algorithm using $b=2$ has a higher output SNR than that using $b=1$. For each scheme, the boundary of the rate-energy region reveals the optimal trade-off between the maximum harvested

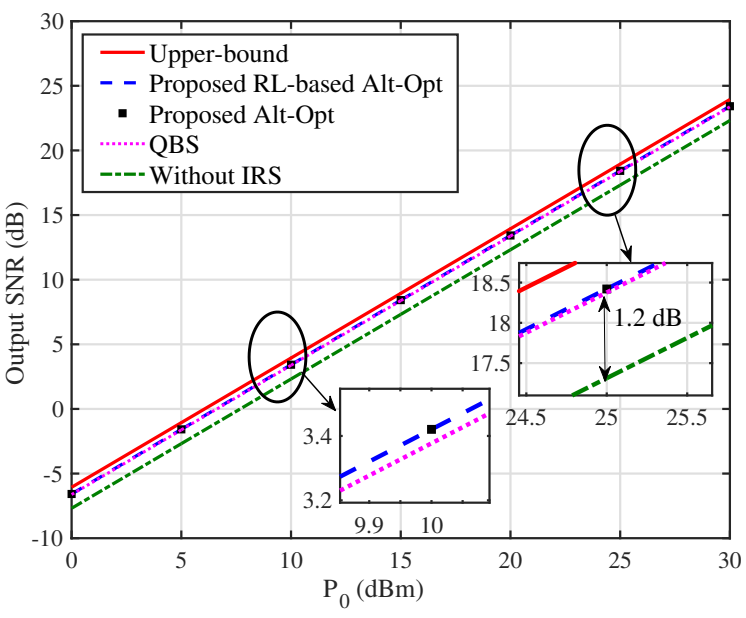

(a) $N_{R}=8$

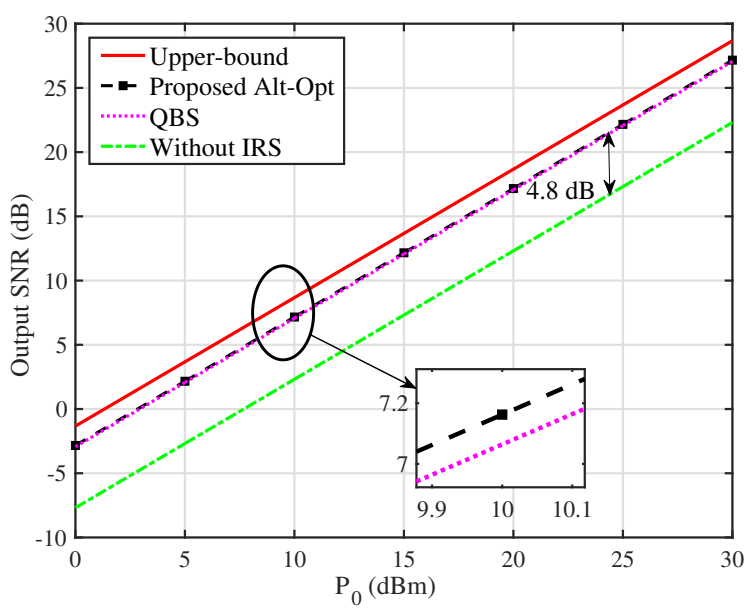

(b) $N_{R}=30$

Fig. 4. Achievable output SNRs as a function of the BS transmit power $P_{0}$ under different IRS setups.

energy and the achieved output SNR. Specifically, we firstly identify two types of boundary points, namely $\left(E_{0}^{\mathrm{mi}}, \gamma_{\max }\right)$ and $\left(E_{0}^{\max }, \gamma_{\mathrm{mi}}\right)$, where the maximum achievable output SNR $\gamma_{\max }$ is obtained from solving problem (11) using the branchand-bound algorithm and the resultant energy threshold is $E_{0}^{\mathrm{mi}}$, while $E_{0}^{\max }$ is derived from solving problem (6) following the idea of alternating optimization and the resultant output SNR is $\gamma_{\mathrm{mi}}=P_{0}\left|\boldsymbol{h}_{I}^{H} \boldsymbol{\mu}\left(\boldsymbol{H}_{E}^{H} \boldsymbol{H}_{E}\right)\right|^{2} / \sigma_{I}^{2}$. Then it is readily found from Fig. 3 (b) that the maximum output SNR is attained provided that $E_{0} \in\left[0, E_{0}^{\mathrm{mi}}\right)$. Conversely, for $E_{0} \in\left[E_{0}^{\mathrm{mi}}, E_{0}^{\max }\right]$, the maximum achievable SNR decreases upon the increase of $E_{0}$. Note that the above conclusions coincide with the feasibility and tightness analysis in Section III-A and Section III-B.

In Fig. 4, we consider two setups of the IRS reflecting elements, denoted by $N_{R}=8$ and $N_{R}=32$ corresponding to small-scale and large-scale systems, respectively, and set $E_{0}=\frac{\left(E_{0}^{\operatorname{mi}}+E_{0}^{\max }\right)}{2} \mathrm{dBm}$ for each considered $P_{0}$. Observe that different values of $P_{0}$ usually correspond to distinct thresholds $E_{0}$, which however guarantees the active energy harvesting constraint for each $P_{0}$. In particular, if problem (5) with $E_{0}=\frac{\left(E_{0}^{\mathrm{mi}}+E_{0}^{\max }\right)}{2} \mathrm{dBm}$ is infeasible when considering both the QBS scheme and the benchmark scheme without IRS, the corresponding output SNR is set to zero. Fig. 4 (a) and Fig. 4 (b) then depict the achievable output SNRs as a function of the BS transmit power $P_{0}$ for different algorithms under the 


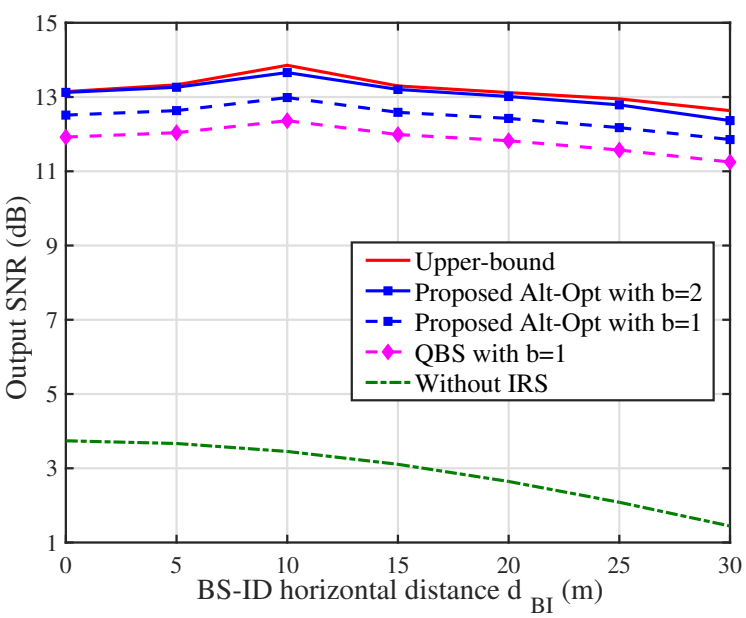

(a) $N_{R}=32$

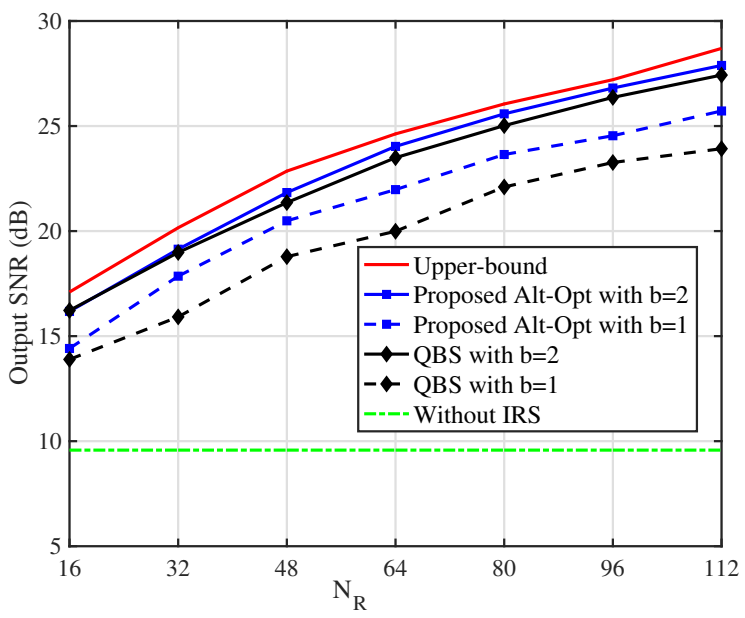

(b) $d_{\mathrm{BI}}=10$

Fig. 5. Achievable output SNR versus: (a) the BS-ID horizontal distance $d_{\mathrm{BI}}$; (b) the number of IRS reflecting elements $N_{R}$.

above two IRS setups, respectively.

In Fig. 4 (a) with $N_{R}=8$, we clearly find that both the proposed Alt-Opt algorithm in Section III-C and the QBS scheme perform close to the proposed RL-based AltOpt algorithm in Section III-B. Moreover, they also approach the upper-bound scheme with continuous phase shifts and outperform the benchmark scheme without IRS by a SNR gain of around $1.2 \mathrm{~dB}$. However, since the QBS scheme utilizes the SDR technique to obtain the optimal IRS continuous phase shifts, it generally has a higher computational complexity than the Alt-Opt algorithm. We also extend this study into a large-scale system (i.e. $N_{R}=32$ ) in Fig. 4 (b), where the proposed RL-based Alt-Opt algorithm is not simulated due to its high complexity caused by the auxillary binary variable of dimension $L^{2} N_{R}\left(N_{R}-1\right) / 2=7936$. Compared to Fig. 4 (a), we firstly observe from Fig. 4 (b) that a larger performance gap between the proposed Alt-Opt algorithm in Section III-C and the upper-bound scheme. This is because the signal directly from the BS and that reflected by the IRS with discrete phase shifts cannot be superimposed constructively at the ID as in the case of continuous phase shifts, hence resulting in certain performance loss (which becomes even worse for a large $N_{R}$ ). Moreover, we find that a higher SNR gain (about $4.8 \mathrm{~dB}$ ) is achieved by both the Alt-Opt algorithm and the QBS scheme over the benchmark scheme without IRS.

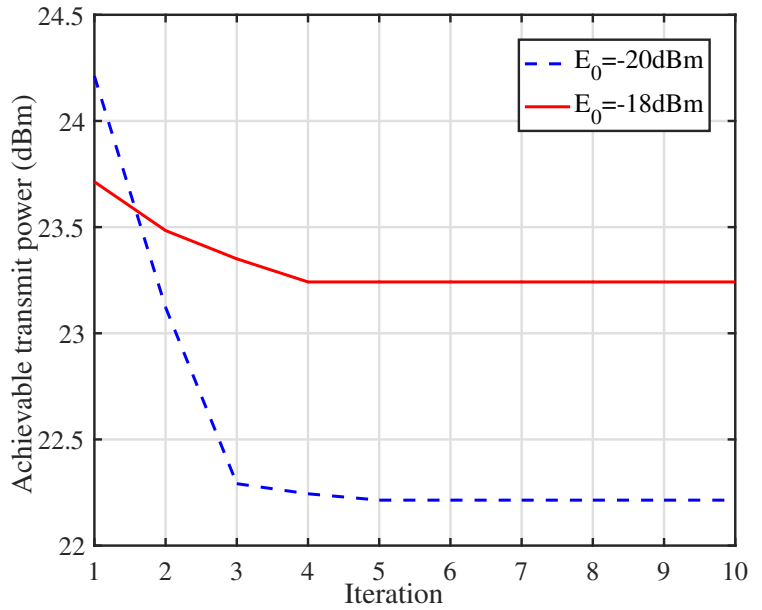

(a) Inner Alt-Opt algorithm

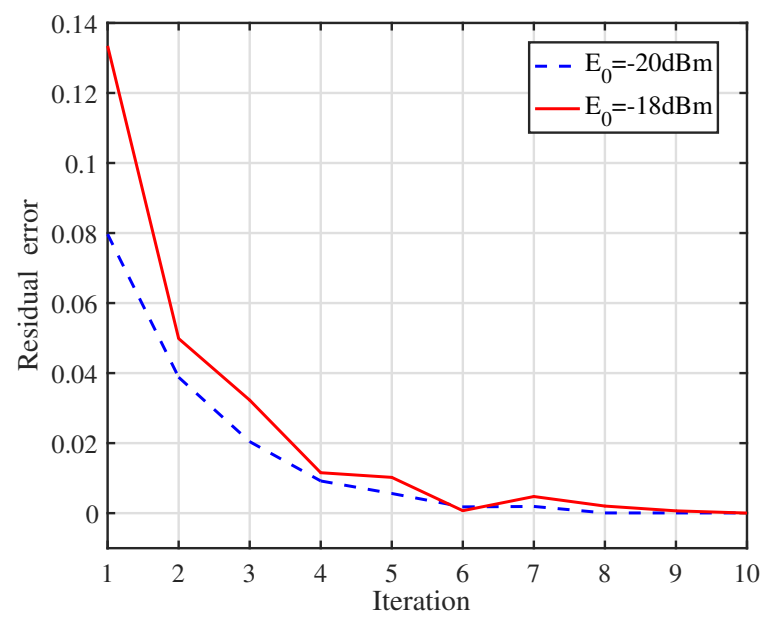

(b) Outer bisection search

Fig. 6. Convergence of the proposed Algorithm 1 for solving problem (P1).

Next, we set $E_{0}=-20 \mathrm{dBm}$ and the location of a single ID as $\left(0, d_{\mathrm{BI}}, 60\right) \mathrm{m}$. Other parameters remain the same as those in Fig. 3. Then Fig. 5 (a) illustrates the achievable output SNRs as a function of the BS-ID horizontal distance $d_{\mathrm{BI}}$ for the proposed Alt-Opt algorithm in Section III-C and benchmarks. We firstly find that all algorithms (except the benchmark scheme without IRS) achieve the highest SNR at $d_{\mathrm{BI}}=10$ $m$ denoting the minimum distance between the ID and IRS. Moreover, due to an expanded set of IRS discrete phase shifts, the Alt-Opt algorithm using $b=2$ clearly realizes better SNR performance than that using $b=1$ and also performs very close to the upper-bound scheme. Nonetheless, the output SNR achieved by the benchmark scheme without IRS decreases with the increasing $d_{\mathrm{BI}}$ followed by severe signal power attenuation. Furthermore, Fig. 5 (b) plots the achieved SNRs versus the number of IRS reflecting elements $N_{R}$ for different algorithms, where $E_{0}=-20 \mathrm{dBm}$ and $d_{\mathrm{BI}}=10$ are set. It is clear that the maximum output SNRs of all schemes increase with $N_{R}$ due to the increasing spatial degrees of freedom offered by the IRS, except the benchmark scheme without IRS. Also, the proposed Alt-Opt algorithm with $b=2$ performs closer to the upper-bound scheme than that with $b=1$.

\section{MIME scenario}

In contrast to the SISE scenario, the max-min SINR among all IDs subject to the minimum total harvested energy at all 
ERs is studied in the MIME scenario. In this subsection, we aim for illustrating the minimum output SINR performance of all proposed algorithms in Section IV. Unless specified otherwise, we set $b=1, N_{R}=48$ and $K_{I}=K_{E}=2$. The locations of multiple ERs and IDs are generated following the simulation setup in Fig. 2 with $r_{E}=0.5$ and $r_{I}=10$. All other parameters are initially chosen as those in Fig. 3 (a).

Fig. 6 firstly shows the convergence of the proposed Algorithm 1 , which consists of a simple outer bisection search and an inner Alt-Opt algorithm between problems (28) and (32). Specifically, we consider two different energy harvesting thresholds, namely $E_{0}=-20 \mathrm{dBm}$ and $E_{0}=-18 \mathrm{dBm}$. It can be observed from Fig. 6 (a) that for both cases, the proposed Alt-Opt algorithm for solving problem (P2) with given $\gamma=5 \mathrm{~dB}$ monotonically converges to the minimum total transmit power after about 8 iterations. Moreover, a higher transmit power is achieved when $E_{0}=-18 \mathrm{dBm}$ compared with the case of $E_{0}=-20 \mathrm{dBm}$, since a large $E_{0}$ generally leads to a small feasible region of problem (P1). Furthermore, Fig. 6(b) shows the convergence of the outer bisection search for the two considered energy thresholds, where the BS transmit power margin, namely $\left|\sum_{i_{I} \in \mathcal{K}_{I}}\left\|\boldsymbol{v}_{i_{I}}\right\|^{2}-P_{0}\right|$, is adopted as a performance metric. Note that the bisection search is not required to find a sequence of monotonically decreasing power margin, its efficiency is demonstrated only when the power margin becomes zero. Using this fact, we can find from Fig. 6 (b) that for each $E_{0}$, the achieved BS transmit power margin approaches zero after about 10 searches.

Like in Fig. 4, we also assume two IRS setups in the MIME scenario given by $N_{R}=8$ in Fig. 7 (a) and Fig. 7 (b) as well as $N_{R}=48$ in Fig. 7 (c), respectively. Moreover, both the inactive and active energy harvesting constraints associated with $E_{0}=E_{0}^{\mathrm{mi}} / 2 \mathrm{dBm}$ and $E_{0}=\left(E_{0}^{\mathrm{mi}}+E_{0}^{\max }\right) / 2$ $\mathrm{dBm}$, respectively, are considered in Fig. 7, where $E_{0}^{\mathrm{mi}}$ and $E_{0}^{\max }$ can be derived by solving problem (P1) without the energy harvesting constraint using Algorithm 1 and solving problem (25) following the idea of alternating optimization, respectively. Specifically, Fig. 7 plots the minimum output SINRs as a function of the BS transmit power $P_{0}$ for different algorithms under the above two IRS setups. We firstly observe from Fig. 7 (a) with $E_{0}=E_{0}^{\mathrm{mi}} / 2$ that all IRS-related schemes achieve a remarkable improvement of the minimum output SINR when compared to the benchmark scheme without IRS. Furthermore, the proposed Algorithm 1 achieves almost the same performance as an exhaustive search, and meanwhile performs close to the upper-bound scheme. It also has better minimum SINR performance than the proposed two-stage scheme, thanks to its iterative nature. Although the QBS scheme performs slightly better than the two-stage scheme in the low-to-medium power regime, it generally has higher computational complexity and suffers from severe performance loss in the high power regime. This is because the strong inter-user interference in the high-power regime cannot be sufficiently suppressed by coarsely quantized phase shifts.

Similar results can also be observed from Fig. 7 (b) with $E_{0}=\left(E_{0}^{\mathrm{mi}}+E_{0}^{\max }\right) / 2$, where the performance advantage of all IRS-related schemes over the scheme without IRS becomes more evident. This is due to the fact that the size

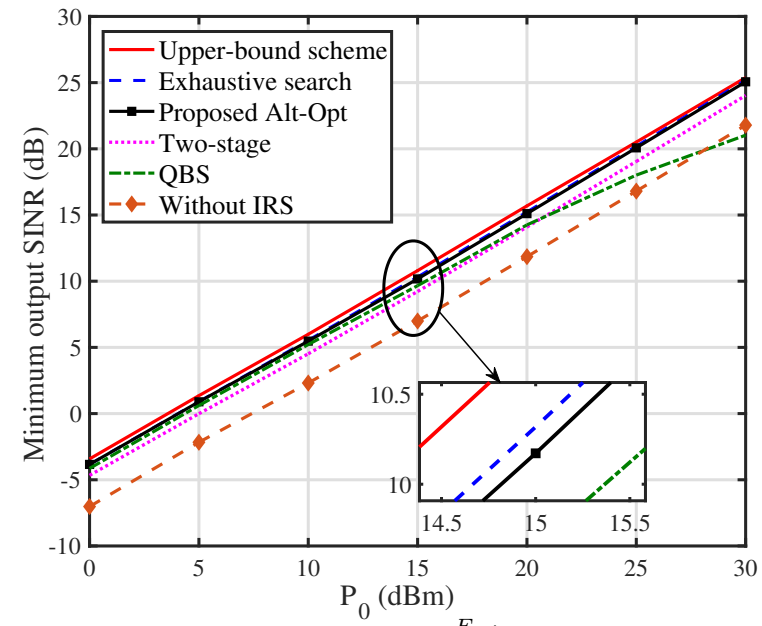

(a) $N_{R}=8, E_{0}=\frac{E_{\mathrm{mi}}}{2} \mathrm{dBm}$

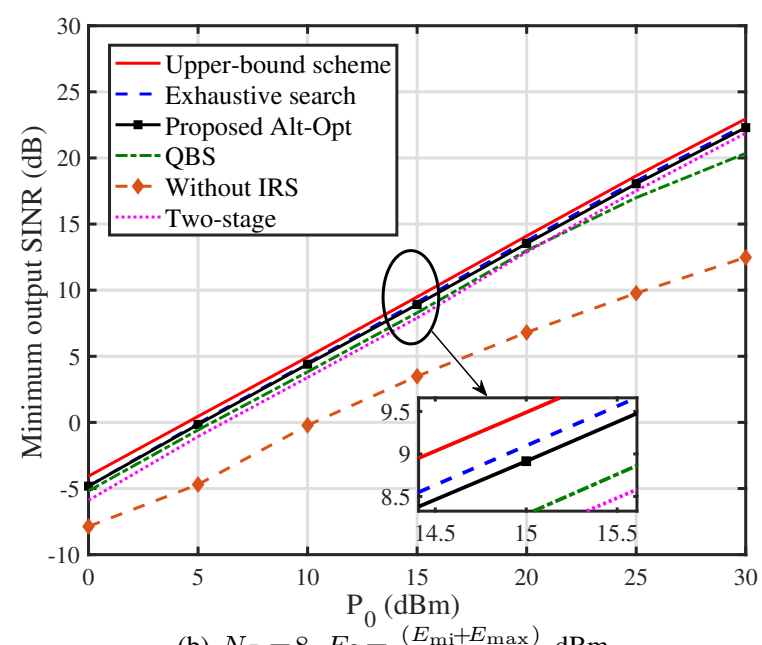

(b) $N_{R}=8, E_{0}=\frac{\left(E_{\mathrm{mi}}+E_{\max }\right)}{2} \mathrm{dBm}$

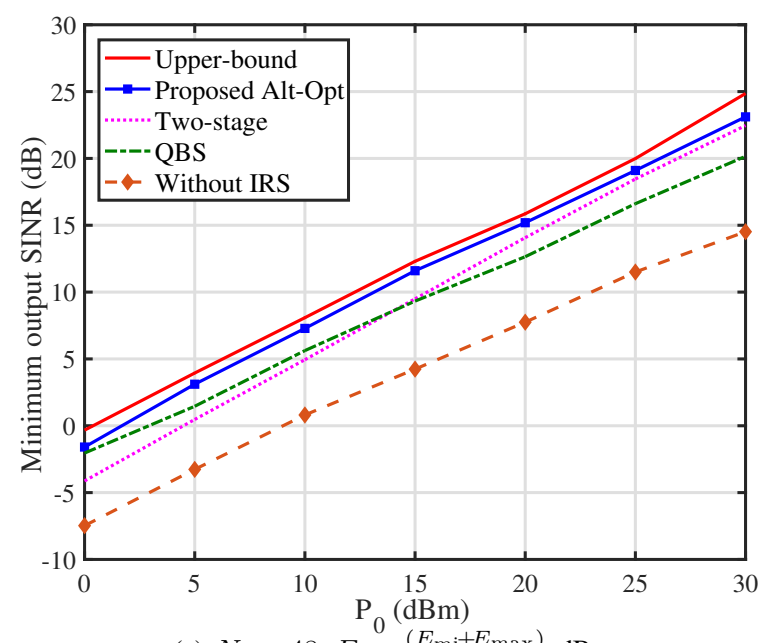

(c) $N_{R}=48, E_{0}=\frac{\left(E_{\mathrm{mi}}+E_{\max }\right)}{2} \mathrm{dBm}$

Fig. 7. Achievable minimum output SINR versus the BS transmit power $P_{0}$ under different parameter setups.

of the feasible region of problem (P1) is largely reduced by the more stringent energy harvesting constraint with $E_{0}=$ $\left(E_{0}^{\mathrm{mi}}+E_{0}^{\mathrm{max}}\right) / 2$, thereby making the advantage of IRS more pronounced. Further, we extend this study to the case of a large number of IRS reflecting elements in Fig. 7 (c) with $E_{0}=\left(E_{0}^{\mathrm{mi}}+E_{0}^{\max }\right) / 2$. It can be seen that the performance gain 


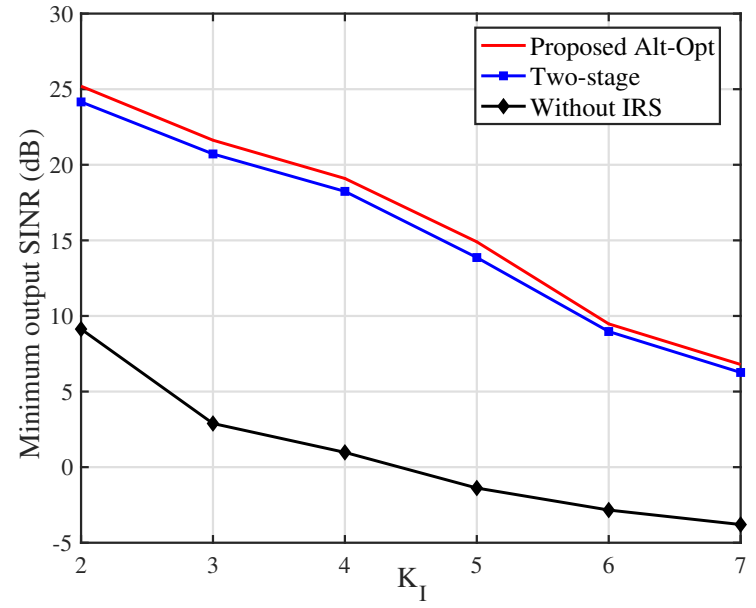

(a) $E_{0}=-20 \mathrm{dBm}$ and $K_{E}=2$

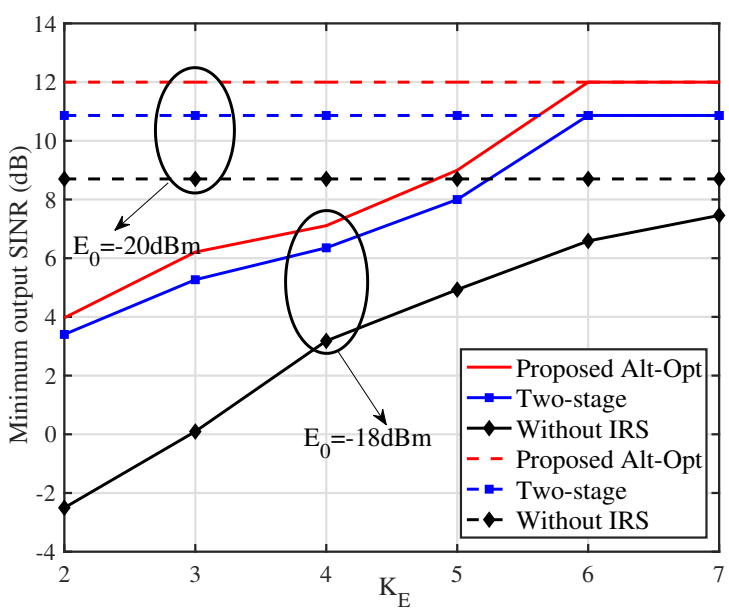

(b) $E_{0}=-20 \mathrm{dBm},-18 \mathrm{dBm}$ and $K_{I}=4$

Fig. 8. Achievable minimum output SINRs versus the number of IDs $K_{I}$ (ERs $K_{E}$ ) for all studied algorithms.

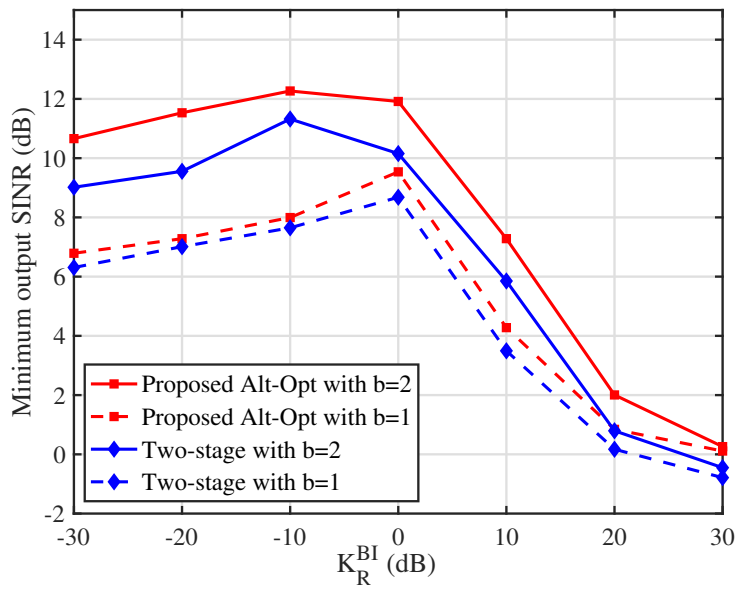

Fig. 9. Achievable minimum output SINRs versus the Rician $K$ factor $K_{\mathrm{R}}^{\mathrm{BI}}$ of the BS-IRS channel for all studied algorithms.

of the proposed Algorithm 1 over all other benchmark schemes is obviously enlarged as compared to that in Fig. 7 (b), because the effectiveness of IRS in cancelling inter-user interference and supporting energy can be significantly improved by a large $N_{R}$. Moreover, in the high-SNR regime, since the achieved output SINR of each ID is dominated by the optimal active BS transmit beamforming attaining both the maximum power transmission and inter-user interference suppression instead of the passive IRS reflection coefficients, which is also the design philosophy of the two-stage scheme, the two-stage scheme achieves a performance close to the proposed Algorithm 1.

Next, by setting $N_{S}=8$, Fig. 8 (a) and Fig. 8 (b) show the minimum output SINRs versus the numbers of IDs $K_{I}$ and ERs $K_{E}$ for different algorithms, respectively. To draw meaningful insights, we consider successively adding one ID within the defined cluster and one ER near the IRS for Fig. 8 (a) and Fig. 8 (b), respectively. Moreover, we set $E_{0}=-20 \mathrm{dBm}$ and $K_{E}=2$. it is clear from Fig. 8 (a) that the minimum output SINRs achieved by all algorithms decrease as the number of IDs $K_{I}$ increases. This is not surprising since our work concentrates on guaranteeing the minimum output SINR performance among all IDs by jointly optimizing the BS transmit beamforming vectors and IRS discrete phase shifts, and the corresponding attainable design freedom is much reduced as $K_{I}$ increases. Moreover, both the proposed Algorithm 1 and two-stage algorithm are superior to the scheme without IRS, which again demonstrates the effectiveness of deploying IRS in the MIME scenario.

Furthermore, Fig. 8(b) plots the minimum output SINRs versus the number of ERs $K_{E}$ for different algorithms, where $K_{I}=4$ and two energy thresholds, i.e. $E_{0}=-20 \mathrm{dBm}$ and $E_{0}=-18 \mathrm{dBm}$, are adopted. For each studied algorithm, we firstly find that for the case of $E_{0}=-20 \mathrm{dBm}$, the achieved minimum output SINR keeps constant regardless of the increasing $K_{E}$, which is due to the inactive energy harvesting constraint. On the other hand, when $E_{0}=-18 \mathrm{dBm}$ is considered, it can be seen that the achieved minimum output SINR firstly increases with $K_{E}$, and then becomes saturated. This can be explained as follows. For a small range of $K_{E}$, the energy harvesting constraint with $E_{0}=-18 \mathrm{dBm}$ is quite likely to be active. In this context, the increasing $K_{E}$ usually leads to the fact that more BS transmit power is reserved for improving the output SINRs of all IDs under the premise of guaranteeing the required total harvested energy. Nevertheless, when $K_{E}$ increases to a large value (i.e. $K_{E}=7$ ) such that the energy harvesting constraint becomes inactive, the highest minimum output SINR is realized and maintained.

Finally, we consider $b=1,2$ and plot the minimum output SINR performance of the proposed Alt-Opt and Two-stage algorithms versus the Rician $K$ factor $K_{\mathrm{R}}^{\mathrm{BI}}$ of the BS-IRS channel. We firstly find that both the two proposed algorithms with $b=2$ achieve a higher minimum output SINR than their respective counterparts with $b=1$. Moreover, the performance gain of the Alt-Opt algorithm over the Two-stage scheme is more evident for $b=2$. For each algorithm, the minimum output SINR performance is sharply reduced for a large $K_{\mathrm{R}}^{\mathrm{BI}}=30$ $\mathrm{dB}$. This is because in the case of small $K_{\mathrm{R}}^{\mathrm{BI}}$, i.e. $K_{\mathrm{R}}^{\mathrm{BI}}=-30$ $\mathrm{dB}$, the BS-IRS channel tends to be Rayleigh distributed and usually leads to low correlation among effective BS-ID channels. In this context, the full spatial multiplexing gain in MIME scenario can be exploited to suppress inter-user interference. Nevertheless, when $K_{\mathrm{BI}}$ increases to $30 \mathrm{~dB}$, the BS-IRS channel becomes dominated by the LoS component, and it is thereby rank-deficient. As such, the effective BS-ID channels also become strongly correlated, hence the inter-user 
interference cannot be effectively suppressed, thus leading to the degraded minimum output SINR performance.

\section{CONCLUSIONS}

In this paper, we investigated the max-min SINR optimization of the IRS-aided MIMO SWIPT system by jointly optimizing the active BS transmit beamforming vectors and the passive IRS reflection coefficients, while guaranteeing the minimum required total harvested energy. Moreover, we considered the IRS with discrete phase shifts from the perspective of practical applications. The resultant combinatorial max-min SINR problem is generally non-convex and computationally intractable. To further explore its inherent properties, we firstly study its feasibility and the tightness of the involved energy harvesting constraint in the SISE scenario. Then for two distinct value ranges of the energy threshold, we proposed the optimal branch-and-bound algorithm and the suboptimal alternating optimization algorithm, respectively. To avoid the high complexity of the LIP for a large system, an elementby-element iterative strategy is also introduced for the IRS reflection coefficients design. Furthermore, we extended our work into the MIME scenario and transformed the intractable max-min SINR problem into an equivalent power minimization problem, which can also be efficiently solved by an alternating optimization algorithm. Moreover, motivated by the monotonicity of the BS transmit power w.r.t the minimum output SINR, the classical bisection search was applied to find a high-quality suboptimal solution to the original max-min SINR problem. To reduce the iteration complexity, we also developed a two-stage scheme. Numerical simulation results demonstrated the convergence and superior performance of the proposed alternating optimization algorithms over the adopted benchmarks.

\section{APPENDIX A}

We can prove this lemma by contradiction. Let's assume $\left\{\boldsymbol{v}_{I}^{\star}, \boldsymbol{\Phi}^{\star}\right\}$ to be the optimal solution of problem (5) for some $E_{0} \in\left[E_{0}^{\operatorname{mi}}, E_{0}^{\max }\right]$. Consider the contrary, i.e. $\eta_{E}\left\|\boldsymbol{H}_{E}^{\star} \boldsymbol{v}_{I}^{\star}\right\|^{2}>$ $E_{0}$, and denote the Lagrangian function of problem (5) given the optimal $\boldsymbol{\Phi}^{\star}$ as

$$
\begin{aligned}
& L\left(\boldsymbol{v}_{I}, \lambda, \beta\right) \\
& =\left|\boldsymbol{h}_{I}^{H} \boldsymbol{v}_{I}\right|^{2}+\lambda\left(P_{0}-\left\|\boldsymbol{v}_{I}\right\|^{2}\right)+\beta\left(\eta_{E}\left\|\boldsymbol{H}_{E} \boldsymbol{v}_{I}\right\|^{2}-E_{0}\right)
\end{aligned}
$$

where $\lambda \geq 0$ and $\beta \geq 0$ denote the optimal dual variables corresponding to the transmit power constraint and the energy harvesting constraint, respectively. Furthermore, the Karush-Kuhn-Tucker (KKT) necessary optimality conditions of problem (5) given the optimal $\Phi^{\star}$ are expressed as

$$
\begin{aligned}
& \left(\boldsymbol{h}_{I} \boldsymbol{h}_{I}^{H}+\beta^{\star} \eta_{E} \boldsymbol{H}_{E}^{H} \boldsymbol{H}_{E}^{\star}\right) \boldsymbol{v}_{I}^{\star}=\lambda^{\star} \boldsymbol{v}_{I}^{\star}, \\
& \boldsymbol{h}_{I} \boldsymbol{h}_{I}^{H}+\beta^{\star} \eta_{E} \boldsymbol{H}_{E}^{H} \boldsymbol{H}_{E} \preceq \lambda^{\star} \boldsymbol{I}_{N_{S}} \\
& \lambda^{\star}\left(\left\|\boldsymbol{v}_{I}^{\star}\right\|^{2}-P_{0}\right)=0, \quad \beta^{\star}\left(\eta_{E}\left\|\boldsymbol{H}_{E}^{\star} \boldsymbol{v}_{I}^{\star}\right\|^{2}-E_{0}\right)=0
\end{aligned}
$$

It follows from $\eta_{E}\left\|\boldsymbol{H}_{E}^{\star} \boldsymbol{v}_{I}^{\star}\right\|^{2}>E_{0}$ and (36c) that $\beta^{\star}=0$. We can also prove $\lambda^{\star}>0$ by contradiction. Assume the contrary, i.e. $\lambda^{\star}=0$, we then have $\boldsymbol{h}_{I} \boldsymbol{h}_{I}^{H} \boldsymbol{v}_{I}^{\star}=\mathbf{0}$ from (36b) and thus $\left|\boldsymbol{h}_{I}^{H} \boldsymbol{v}_{I}\right|=0$ holds, which contradicts to the optimization objective of maximizing $\left|\boldsymbol{h}_{I}^{H} \boldsymbol{v}_{I}\right|$. As a result, $\left\|\boldsymbol{v}_{I}^{\star}\right\|^{2}=P_{0}$ holds due to (36c). The uniquely optimal
$\boldsymbol{v}_{I}^{\star}=\sqrt{P_{0}} \boldsymbol{h}_{I} /\left\|\boldsymbol{h}_{I}\right\|$ can be further derived based on $\beta^{\star}=0$ and (36b). Accordingly, the harvested energy at the ER is given by $\eta_{E} P_{0}\left\|\boldsymbol{H}_{E}^{\star} \boldsymbol{h}_{I}^{\star}\right\|^{2} /\left\|\boldsymbol{h}_{I}^{\star}\right\|^{2}=E_{0}^{\mathrm{mi}}>E_{0}$. This is in contradiction to the initial condition of $E_{0} \in\left[E_{0}^{\mathrm{mi}}, E_{0}^{\max }\right]$, hence Lemma 1 must hold. This completes the proof.

\section{APPENDIX B}

For any given $\boldsymbol{\Phi}$, let's recall the Lagrangian function of problem (5), as shown in (35), we then have the following dual problem

$$
\min _{\lambda, \beta} \max _{\boldsymbol{v}_{I}} L\left(\boldsymbol{v}_{I}, \lambda, \beta\right), \quad \text { s.t. } \lambda \geq 0, \beta \geq 0 .
$$

According to [32], it is known that the dual problem (37) is always convex. As a result, in order to guarantee the upperbounded dual function $L\left(\boldsymbol{v}_{I}, \lambda, \beta\right)$ in terms of the unconstrained $\boldsymbol{v}_{I}$, we must have $\boldsymbol{h}_{I} \boldsymbol{h}_{I}^{H}+\beta \eta_{E} \boldsymbol{H}_{E}^{H} \boldsymbol{H}_{E}-\lambda \boldsymbol{I} \preceq \mathbf{0}$, based on which the dual problem (37) reduces to

$$
\begin{array}{ll}
\min _{\lambda, \beta} & \lambda P_{0}-\beta E_{0}, \\
\text { s.t. } & \boldsymbol{h}_{I} \boldsymbol{h}_{I}^{H}+\beta \eta_{E} \boldsymbol{H}_{E}^{H} \boldsymbol{H}_{E} \preceq \lambda \boldsymbol{I}, \quad \lambda \geq 0, \beta \geq 0 .
\end{array}
$$

Based on the Lagrange dual theory, the optimal objective value $G\left(\lambda^{\star}, \beta^{\star}\right)=\lambda^{\star} P_{0}-\beta^{\star} E_{0}$ of problem (38) actually provides an upper bound on that of problem (5), i.e. $\left|\boldsymbol{h}_{I}^{H} \boldsymbol{v}_{I}^{\star}\right|^{2} \leq$ $G\left(\lambda^{\star}, \beta^{\star}\right)$. By recalling the KKT necessary optimality conditions of problem (5) in (36), we can prove $\lambda^{\star}>0$ and $\left\|\boldsymbol{v}_{I}^{\star}\right\|^{2}=P_{0}$ as in Appendix A. Based on this fact, multiplying both sides of (36a) by $\left(\boldsymbol{v}_{I}^{\star}\right)^{H}$ further yields $\left|\boldsymbol{h}_{I}^{H} \boldsymbol{v}_{I}^{\star}\right|^{2}=\lambda^{\star} P_{0}-$ $\beta^{\star} \eta_{E}\left\|\boldsymbol{H}_{E} \boldsymbol{v}_{I}^{\star}\right\|^{2}=G\left(\lambda^{\star}, \beta^{\star}\right)$, where the last equality holds due to $\eta_{E}\left\|\boldsymbol{H}_{E} \boldsymbol{v}_{I}^{\star}\right\|^{2}=E_{0}$ when $E_{0} \in\left[E_{0}^{\operatorname{mi}}, E_{0}^{\max }\right]$, as proved in Lemma 1. The equality $\left|\boldsymbol{h}_{I}^{H} \boldsymbol{v}_{I}^{\star}\right|^{2}=G\left(\lambda^{\star}, \beta^{\star}\right)$ indicates that the strong duality holds for the nonconvex problem (5) with any given $\boldsymbol{\Phi}$. As a result, we can conclude that the optimal $\boldsymbol{v}_{I}^{\star}$ is actually a dominated eigenvector of $\boldsymbol{h}_{I} \boldsymbol{h}_{I}^{H}+\beta^{\star} \eta_{E} \boldsymbol{H}_{E}^{H} \boldsymbol{H}_{E}$ associated with its maximum eigenvalue $\lambda^{\star}$. Moreover, since the dual problem (38) is always convex, the subgradient method can be applied to obtain the optimal $\left\{\lambda^{\star}, \beta^{\star}\right\}$. This completes the proof.

\section{APPENDIX C}

Let's define the optimal solution of problem (P1) leading to the optimal objective value $\gamma^{\star}\left(P_{0}, E_{0}\right)$ as $\left\{\boldsymbol{v}_{i_{I}}^{1}, \boldsymbol{\Phi}^{1}\right\}$, while $\left\{\boldsymbol{v}_{i_{I}}^{2}, \boldsymbol{\Phi}^{2}\right\}$ denotes the optimal solution of problem (P2) with $\gamma_{0}=\gamma^{\star}\left(P_{0}, E_{0}\right)$ and the corresponding optimal objective value is defined as $P^{\star}\left(\gamma_{0}, E_{0}\right)$. We then prove $P_{0}=P^{\star}\left(\gamma^{\star}\left(P_{0}, E_{0}\right), E_{0}\right)$ by contradiction. Suppose the contrary, i.e. $P_{0} \neq P^{\star}\left(\gamma^{\star}\left(P_{0}, E_{0}\right), E_{0}\right)$, the two cases $P_{0}<$ $P^{\star}\left(\gamma^{\star}\left(P_{0}, E_{0}\right), E_{0}\right)$ and $P_{0}>P^{\star}\left(\gamma^{\star}\left(P_{0}, E_{0}\right), E_{0}\right)$ are then discussed, respectively.

Firstly, it is noted that the optimal $\boldsymbol{v}_{i_{I}}^{1}$ 's of problem (P1) must satisfy $\sum_{i_{I} \in \mathcal{K}_{I}}\left\|\boldsymbol{v}_{i_{I}}^{1}\right\|^{2}=P_{0}$, which can be easily proved by contradiction. We find that the case of $P_{0}<$ $P^{\star}\left(\gamma^{\star}\left(P_{0}, E_{0}\right), E_{0}\right)$ clearly contradicts the optimality of $\left\{\boldsymbol{v}_{i_{I}}^{2}, \boldsymbol{\Phi}^{2}\right\}$ for problem (P2), since the optimal $\left\{\boldsymbol{v}_{i_{I}}^{1}, \boldsymbol{\Phi}^{1}\right\}$ to problem (P1) is also feasible to problem (P2) with $\gamma_{0}=$ $\gamma^{\star}\left(P_{0}, E_{0}\right)$ and provides a smaller objective value $P_{0}$. Secondly, for the case of $P_{0}>P^{\star}\left(\gamma^{\star}\left(P_{0}, E_{0}\right), E_{0}\right)$, it is clear that the optimal $\left\{\boldsymbol{v}_{i_{I}}^{2}, \boldsymbol{\Phi}^{2}\right\}$ to problem (P2) is also feasible 
to problem (P1) and the resultant power consumption being less than $P_{0}$. Moreover, considering that $\operatorname{SINR}\left(\left\{c \boldsymbol{v}_{i_{I}}^{2}, \boldsymbol{\Phi}^{2}\right\}\right) \geq$ $\operatorname{SINR}\left(\left\{\boldsymbol{v}_{i_{I}}^{2}, \boldsymbol{\Phi}^{2}\right\}\right), \forall i_{I} \in \mathcal{K}_{I}$ for some $c \geq 1$, we can always find a constant $c>1$ so that $\left\{c \boldsymbol{v}_{i_{I}}^{2}, \Phi^{2}\right\}$ is also feasible to problem (P1) and simultaneously leads to a larger value of the minimum output SINR. This is a contradiction to the optimality of $\left\{\boldsymbol{v}_{i_{I}}^{1}, \boldsymbol{\Phi}^{1}\right\}$ for problem (P1). As a result, $P_{0}=P^{\star}\left(\gamma^{\star}\left(P_{0}, E_{0}\right), E_{0}\right)$ must hold.

Next, we prove (27) by contradiction. Specifically, the optimal solutions of problem (P2) corresponding to the optimal objective values $P^{\star}\left(\gamma_{0}^{1}, E_{0}\right)$ and $P^{\star}\left(\gamma_{0}^{2}, E_{0}\right)$ with $\gamma_{0}^{1}<\gamma_{0}^{2}$ are denoted by $\left\{\tilde{\boldsymbol{v}}_{i_{I}}^{1}, \boldsymbol{\Phi}_{1}\right\}$ and $\left\{\tilde{\boldsymbol{v}}_{i_{I}}^{2}, \boldsymbol{\Phi}_{2}\right\}$, respectively. Clearly, $\left\{\tilde{\boldsymbol{v}}_{i_{I}}^{2}, \boldsymbol{\Phi}_{2}\right\}$ is also feasible to problem (P2) with $\gamma_{0}=\gamma_{0}^{1}$, and thus we have $P^{\star}\left(\gamma_{0}^{2}, E_{0}\right) \geq P^{\star}\left(\gamma_{0}^{1}, E_{0}\right)$. Moreover, the optimal objective value $P^{\star}\left(\gamma_{0}, E_{0}\right)$ is observed to be continuous in arguments $\left\{\gamma_{0}, E_{0}\right\}$, since both the quadratic objective function and constraints of problem (P2) are continuous and the set of feasible solution is closed and bounded. As such, we can conclude that $P^{\star}\left(\gamma_{0}, E_{0}\right)$ is monotonically non-decreasing w.r.t $\gamma_{0}$. This completes the proof.

\section{APPENDIX D}

Recall [33, Lemma 3.1], the separable SDP problem (28) with $1+K_{I}$ linear constraints always admits an optimal solution $\boldsymbol{V}_{i_{I}}^{\star}$ 's such that $\sum_{i_{I} \in \mathcal{K}_{I}}\left(\operatorname{rank}\left(\boldsymbol{V}_{i_{I}}^{\star}\right)\right)^{2} \leq 1+K_{I}$, based on which we have $\operatorname{rank}\left(\boldsymbol{V}_{i_{I}}^{\star}\right)=1, \forall i_{I} \in \mathcal{K}_{I}$ to meet individual SINR constraints. However, this theorem only shows the existence of the optimal rank-1 solution to problem (28). Next, we prove that the optimal solution of problem (28) is rank-1 with probability one by using its KKT necessary and sufficient conditions, i.e.

$$
\begin{aligned}
& \boldsymbol{Q}_{i_{I}}^{\star}-\lambda_{i_{I}}^{\star} \boldsymbol{h}_{i_{I}} \boldsymbol{h}_{i_{I}}^{H}=\boldsymbol{Z}_{i_{I}}^{\star}, \\
& \boldsymbol{Q}_{i_{I}}^{\star}=\boldsymbol{I}_{N_{S}}-\beta^{\star} \sum_{i_{E} \in \mathcal{K}_{E}} \eta_{i_{E}} \boldsymbol{H}_{i_{E}}^{H} \boldsymbol{H}_{i_{E}}+\sum_{j_{I} \neq i_{I}} \lambda_{j_{I}}^{\star} \boldsymbol{h}_{j_{I}} \boldsymbol{h}_{j_{I}}^{H}, \\
& \boldsymbol{Z}_{i_{I}}^{\star} \boldsymbol{V}_{i_{I}}^{\star}=\mathbf{0}_{N_{S}}, \quad \forall i_{I} \in \mathcal{K}_{I},
\end{aligned}
$$

where $\beta^{\star} \geq 0, \lambda_{i_{I}}^{\star} \geq 0$ and $\boldsymbol{Z}_{i_{I}}^{\star} \succeq \mathbf{0}$ denote the optimal dual variables associated with constraints (28b), (28c) and $\boldsymbol{V}_{i_{I}} \succeq \mathbf{0}$, respectively. It follows from (39a) and (39c) that $\left(\boldsymbol{Q}_{i_{I}}^{\star}-\lambda_{i_{I}}^{\star} \boldsymbol{h}_{i_{I}} \boldsymbol{h}_{i_{I}}^{H}\right) \boldsymbol{V}_{i_{I}}^{\star}=\mathbf{0}_{N_{S}}, \forall i_{I} \in \mathcal{K}_{I}$. Next, two cases of $\beta^{\star}$, i.e. $\beta^{\star}=0$ and $\beta^{\star}>0$, are studied to prove the rank-1 optimality of problem (28c). Firstly, for the case of $\beta^{\star}=0$, we naturally have $\boldsymbol{Q}_{i_{I}}^{\star} \succ \mathbf{0}, \forall i_{I} \in \mathcal{K}_{I}$. Further, based on $\left(\boldsymbol{Q}_{i_{I}}^{\star}-\lambda_{i_{I}}^{\star} \boldsymbol{h}_{i_{I}} \boldsymbol{h}_{i_{I}}^{H}\right) \boldsymbol{V}_{i_{I}}^{\star}=\mathbf{0}_{N_{S}}$ and $\boldsymbol{V}_{i_{I}}^{\star} \neq \mathbf{0}$ required for meeting the SINR requirement of ID $i_{I}$, we have $\operatorname{rank}\left(\boldsymbol{V}_{i_{I}}^{\star}\right)=$ $\operatorname{rank}\left(\boldsymbol{Q}_{i_{I}}^{\star} \boldsymbol{V}_{i_{I}}^{\star}\right)=\operatorname{rank}\left(\lambda_{i_{I}}^{\star} \boldsymbol{h}_{i_{I}} \boldsymbol{h}_{i_{I}}^{H} \boldsymbol{V}_{i_{I}}^{\star}\right)=1, \forall i_{I} \in \mathcal{K}_{I}$.

Secondly, for the case of $\beta^{\star}>0$, under the general assumption of independently distributed channels $\boldsymbol{h}_{i_{I}}$ 's and $\boldsymbol{H}_{i_{E}}$ 's, it follows from (39b) that $\operatorname{rank}\left(\boldsymbol{Q}_{i_{I}}^{\star}\right) \geq N_{S}-1, \forall i_{I} \in \mathcal{K}_{I}$, with probability one [34]. Using this fact, on the one hand, it can be inferred from (39a) and (39b) that for some $i_{I} \in \mathcal{K}_{I}$ with $\lambda_{i_{I}}^{\star}=0$, we have $Z_{i_{I}}^{\star}=\boldsymbol{Q}_{i_{I}}^{\star}$ and $\operatorname{rank}\left(\boldsymbol{Q}_{i_{I}}^{\star}\right)=N_{S}-1$ to ensure the nonzero $\boldsymbol{V}_{i_{I}}^{\star}$. Correspondingly, the rank-1 optimal $\boldsymbol{V}_{i_{I}}^{\star}$ can be obtained. On the other hand, for some $\bar{i}_{I} \in \mathcal{K}_{I}$ with $\lambda_{\bar{i}_{I}}^{\star}>0, \operatorname{rank}\left(\boldsymbol{Q}_{\bar{i}_{I}}^{\star}\right)=N_{S}$ holds with probability one. Otherwise, by jointly referring to $\boldsymbol{Z}_{i_{I}}^{\star} \succeq \mathbf{0}$ and (39a), it yields $\boldsymbol{Q}_{\bar{i}_{I}}^{\star} \succeq \lambda_{\bar{i}_{I}} \boldsymbol{h}_{\bar{i}_{I}} \boldsymbol{h}_{\bar{i}_{I}}^{H}$ implying the null space of $\boldsymbol{Q}_{\bar{i}_{I}}^{\star}$, i.e.
$\operatorname{Null}\left(\boldsymbol{Q}_{\bar{i}_{I}}^{\star}\right)$ which is rank-1 due to $\operatorname{rank}\left(\boldsymbol{Q}_{\bar{i}_{I}}^{\star}\right) \geq N_{S}-1$, also lies in that of $\boldsymbol{h}_{\bar{i}_{I}} \boldsymbol{h}_{\bar{i}_{I}}^{H}$. Recall (39b), since the eigenspace of $\boldsymbol{Q}_{\bar{i}_{I}}^{\star}$ is uniquely and jointly determined by the independently distributed channels $\boldsymbol{h}_{i_{I}}$ 's and $\boldsymbol{H}_{i_{E}}$ 's, it is almost impossible to realize $\boldsymbol{h}_{\bar{i}_{I}}^{H} \operatorname{Null}\left(\boldsymbol{Q}_{\bar{i}_{I}}^{\star}\right) \boldsymbol{h}_{\bar{i}_{I}}=0, \forall \bar{i}_{I} \in \mathcal{K}_{I}$. As such, $\operatorname{rank}\left(\boldsymbol{Q}_{\bar{i}_{I}}^{\star}\right)=N_{S}$ can be established with probability one. Furthermore, the rank-1 optimal $\boldsymbol{V}_{\bar{i}_{I}}^{\star}$ can also be proved for $\beta^{\star}>0$, similarly to the case of $\beta^{\star}=0$. Overall, we finally conclude that the optimal solution of the convex problem (28) satisfies $\operatorname{rank}\left(\boldsymbol{V}_{i_{I}}^{\star}\right)=1, \forall i_{i} \in \mathcal{K}_{I}$. This proof is completed.

\section{REFERENCES}

[1] L. Lu, G. Y. Li, A. L. Swindlehurst, A. Ashikhmin, and R. Zhang, "An overview of massive MIMO: Benefits and challenges," IEEE J. Sel. Top. Sign. Proces., vol. 8, no. 5, pp. 742-758, 2014.

[2] H. Liu, F. Hu, S. Qu et al., "Multipoint wireless information and power transfer to maximize sum-throughput in WBAN with energy harvesting," IEEE Internet Things J., vol. 6, no. 4, pp. 7069-7078, 2019.

[3] L. O. Varga, G. Romaniello, Vučinić et al., "Greennet: An energyharvesting IP-enabled wireless sensor network," IEEE Internet Things J., vol. 2, no. 5, pp. 412-426, 2015.

[4] G. Yu, X. Chen, C. Zhong et al., "Design, analysis and optimization of a large intelligent reflecting surface aided B5G cellular Internet of Things," IEEE Internet Things J., 2020.

[5] Q. Wu and R. Zhang, "Towards smart and reconfigurable environment: Intelligent reflecting surface aided wireless network," IEEE Commun. Mag., 2019.

[6] — "Intelligent reflecting surface enhanced wireless network via joint active and passive beamforming," IEEE Trans. Wireless Commun., vol. 18, no. 11, pp. 5394-5409, 2019.

[7] Q. U. A. Nadeem, A. Kammoun, A. Chaaban, M. Debbah, and M.S. Alouini, "Intelligent reflecting surface assisted multi-user MISO communication," arXiv preprint arXiv:1906.02360, 2019.

[8] S. Zhang and R. Zhang, "Capacity characterization for intelligent reflecting surface aided MIMO communication," IEEE J. Sel. Areas in Commun., 2020.

[9] C. Pan, H. Ren, K. Wang, W. Xu, M. Elkashlan, A. Nallanathan, and L. Hanzo, "Intelligent reflecting surface for multicell MIMO communications," arXiv preprint arXiv:1907.10864, 2019.

[10] Y. Yang, B. Zheng, S. Zhang, and R. Zhang, "Intelligent reflecting surface meets OFDM: Protocol design and rate maximization," IEEE Trans. Commun., 2020.

[11] B. Zheng and R. Zhang, "Intelligent reflecting surface-enhanced OFDM: Channel estimation and reflection optimization," IEEE Wireless Commun. Lett., 2019.

[12] Z. Q. He and X. Yuan, "Cascaded channel estimation for large intelligent metasurface assisted massive MIMO," IEEE Wireless Commun. Lett. 2019.

[13] C. You, B. Zheng, and R. Zhang, "Progressive channel estimation and passive beamforming for intelligent reflecting surface with discrete phase shifts," arXiv preprint arXiv:1912.10646, 2019.

[14] X. Lu, P. Wang, D. Niyato, D. I. Kim, and Z. Han, "Wireless networks with RF energy harvesting: A contemporary survey," IEEE Commun. Surveys Tuts., vol. 17, no. 2, pp. 757-789, 2014.

[15] Y. Huang, M. Liu, and Y. Liu, "Energy-efficient SWIPT in IoT distributed antenna systems," IEEE Internet Things J., vol. 5, no. 4, pp. 2646-2656, 2018.

[16] S. H. Chae, C. Jeong, and S. H. Lim, "Simultaneous wireless information and power transfer for Internet of Things sensor networks," IEEE Internet Things J., vol. 5, no. 4, pp. 2829-2843, 2018.

[17] Q. Wu and R. Zhang, "Weighted sum power maximization for intelligent reflecting surface aided SWIPT," IEEE Wireless Commun. Lett., 2019.

[18] Y. Tang, G. Ma, H. Xie, J. Xu, and X. Han, "Joint transmit and reflective beamforming design for IRS-assisted multiuser MISO SWIPT systems," arXiv preprint arXiv:1910.07156, 2019.

[19] Q. Wu and R. Zhang, "Joint active and passive beamforming optimization for intelligent reflecting surface assisted SWIPT under QoS constraints," arXiv preprint arXiv:1910.06220, 2019.

[20] X. Mu, Y. Liu, L. Guo, J. Lin, and N. Al-Dhahir, "Exploiting intelligent reflecting surfaces in multi-antenna aided NOMA systems," arXiv preprint arXiv:1910.13636, 2019. 
[21] B. Zheng, Q. Wu, and R. Zhang, "Intelligent reflecting surface-assisted multiple access with user pairing: NOMA or OMA?" IEEE Commun. Lett., 2020.

[22] X. Guan, Q. Wu, and R. Zhang, "Intelligent reflecting surface assisted secrecy communication: Is artificial noise helpful or not?" IEEE Wireless Commun. Lett., 2020

[23] M. Cui, G. Zhang, and R. Zhang, "Secure wireless communication via intelligent reflecting surface," IEEE Wireless Commun. Lett., vol. 8, no. 5, pp. 1410-1414, 2019.

[24] Q. Wu and R. Zhang, "Beamforming optimization for wireless network aided by intelligent reflecting surface with discrete phase shifts," IEEE Trans. Commun., 2019.

[25] S. Burer and A. N. Letchford, "Non-convex mixed-integer nonlinear programming: A survey," Surv. Oper. Res. Manag. Sci., vol. 17, no. 2 , pp. $97-106,2012$.

[26] P. M. Hahn, B. J. Kim, Guignard et al., "An algorithm for the generalized quadratic assignment problem," Comput. Optim. Appl., vol. 40, no. 3, p. 351, 2008.

[27] L. T. Landau and R. C. de Lamare, "Branch-and-bound precoding for multiuser MIMO systems with 1-bit quantization," IEEE Wireless Commun. Lett., vol. 6, no. 6, pp. 770-773, 2017.

[28] B. Ghojogh, F. Karray, and M. Crowley, "Eigenvalue and generalized eigenvalue problems: Tutorial," arXiv preprint arXiv:1903.11240, 2019.

[29] M. Razaviyayn, M. Hong, and Z.-Q. Luo, "A unified convergence analysis of block successive minimization methods for nonsmooth optimization," SIAM J. Optim., vol. 23, no. 2, pp. 1126-1153, 2013.

[30] L. Wu, P. Babu, and D. P. Palomar, "Transmit waveform/receive filter design for MIMO radar with multiple waveform constraints," IEEE Trans. Signal Process., vol. 66, no. 6, pp. 1526-1540, 2017.

[31] A. Ben-Tal and A. Nemirovski, Lectures on modern convex optimization: Analysis, algorithms, and engineering applications. Siam, 2001, vol. 2.

[32] S. Boyd and L. Vandenberghe, Convex optimization. Cambridge university press, 2004.

[33] Y. Huang and D. P. Palomar, "Rank-constrained separable semidefinite programming with applications to optimal beamforming," IEEE Trans. Signal Process., vol. 58, no. 2, pp. 664-678, 2009.

[34] J. Xu, L. Liu, and R. Zhang, "Multiuser MISO beamforming for simultaneous wireless information and power transfer," IEEE Trans. Signal Process., vol. 62, no. 18, pp. 4798-4810, 2014.

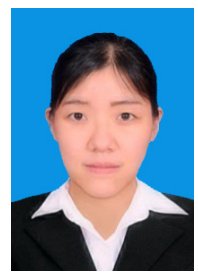

Shiqi Gong (S'14) received the B.S. and Ph.D. degrees in electronic engineering from the Beijing Institute of Technology, Beijing, China, in 2014 and 2020, respectively. She is currently a Postdoctoral Fellow with the State Key Laboratory of Internet of Things for Smart City, University of Macau, Taipa, Macao, China. Her research interests are in the area of signal processing, physical-layer security, resource allocation, and convex optimization.

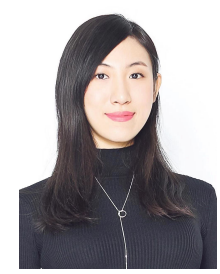

Ziyi Yang received the B.S. degree in electronic engineering from Beijing Institute of Technology, Beijing, China, in 2015. She is working toward the Ph.D. degree in the School of Electronic and Information, Beijing Institute of Technology. Her research interests include signal processing, convex optimization, massive MIMO systems and wireless communications.

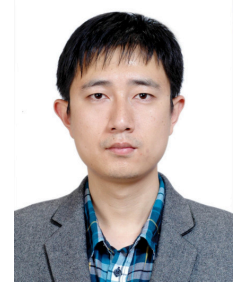

Chengwen Xing (S'08-M'10) received the B.Eng. degree from Xidian University, Xi'an, China, in 2005, and the Ph.D. degree from the University of Hong Kong, Hong Kong, China, in 2010. Since September 2010, he has been with the School of Information and Electronics, Beijing Institute of Technology, Beijing, China, where he is currently a Full Professor. From September 2012 to December 2012, he was a visiting scholar at the University of Macau. His current research interests include statistical signal processing, convex optimization, multivariate statistics, combinatorial optimization, massive MIMO systems, and high frequency band communication systems. Prof. Xing is an Associate Editor for the IEEE Transactions On Vehicular Technology, KSII Transactions on Internet and Information Systems, Transactions on Emerging Telecommunications Technologies, and China Communications.

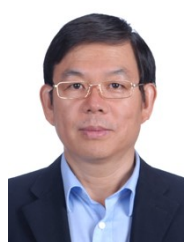

Jianping An (Member, IEEE) received the Ph.D degree from the Beijing Institute of Technology, China, in 1996. He joined the School of Information and Electronics, Beijing Institute of Technology, in 1995, where he is currently a Full Professor and the Dean. His research interests are in the field of digital signal processing, cognitive radio, wireless networks, and high-dynamic broadband wireless transmission technology. Dr. An has received two national awards for technological inventions and science and technology progress.

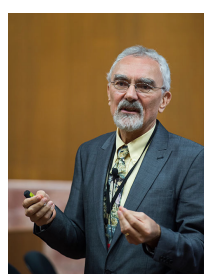

Lajos Hanzo (FIEEE'04, Fellow of the Royal Academy of Engineering F(REng), of the IET and of EURASIP), received his Master degree and Doctorate in 1976 and 1983, respectively, from the Technical University (TU) of Budapest. He was also awarded the Doctor of Sciences (DSc) degree by the University of Southampton (2004) and Honorary Doctorates by the TU of Budapest (2009) and by the University of Edinburgh (2015). He is a Foreign Member of the Hungarian Academy of Sciences and a former Editor-in-Chief of the IEEE Press. He has served several terms as Governor of both IEEE ComSoc and of VTS. He has published 1900+ contributions at IEEE Xplore, 19 Wiley-IEEE Press books and has helped the fast-track career of $123 \mathrm{PhD}$ students. Over 40 of them are Professors at various stages of their careers in academia and many of them are leading scientists in the wireless industry. 